\title{
Méta-chaîne opératoire et transmission culturelle
}

Meta-operational Sequence and Cultural Transmission

\section{Eric Ripoll}

\section{(2) OpenEdition}

\section{Journals}

Édition électronique

URL : https://journals.openedition.org/tc/4909

DOI : $10.4000 /$ tc.4909

ISSN : 1952-420X

Éditeur

Éditions de l'EHESS

\section{Édition imprimée}

Date de publication : 1 décembre 2009

Pagination : 352-375

ISBN : 978-2-7351-1301-9

ISSN : 0248-6016

\section{Référence électronique}

Eric Ripoll, « Méta-chaîne opératoire et transmission culturelle », Techniques \& Culture [En ligne], 52-53 | 2009, mis en ligne le 01 août 2012, consulté le 29 septembre 2022. URL : http://

journals.openedition.org/tc/4909; DOI : https://doi.org/10.4000/tc.4909

Ce document a été généré automatiquement le 29 septembre 2022

Tous droits réservés 


\section{Méta-chaîne opératoire et transmission culturelle}

Meta-operational Sequence and Cultural Transmission

Eric Ripoll

\section{NOTE DE L'ÉDITEUR}

Riche de son expérience d'instructeur paramoteur, Eric Ripoll analyse dans cet article l'apparition de chaînes opératoires atypiques, « des méta-chaînes opératoires ", vecteurs privilégiés d'une culture de la sécurité. 


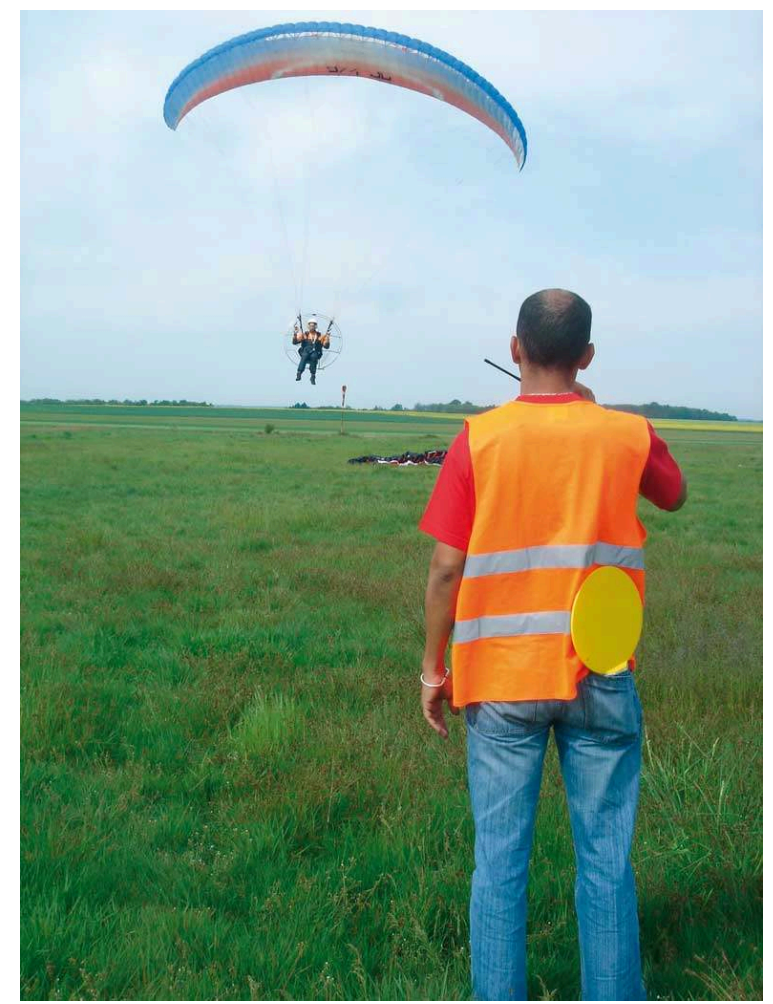

(C) E. Ripoll

"Bonjour, j'espère que je vais apprendre quelque chose...». Cet apprenti-instructeur paramoteur ne fait guère preuve de modestie, pour le premier jour de sa formation! Je constate très vite qu'il maîtrise les « chaînes opératoires » du pilote et une bonne partie de celles de l'instructeur. Pourtant, le soir de ce premier jour : «Au revoir. J'ai été c... ce matin ". Qu'est-ce que ce stagiaire - peu diplomate mais honnête - a bien pu découvrir en quelques heures, qui ne fait partie ni des chaînes opératoires du pratiquant ni de celles du formateur? C'est ce que nous allons tenter de mettre en évidence dans ce bref article ${ }^{1}$. 


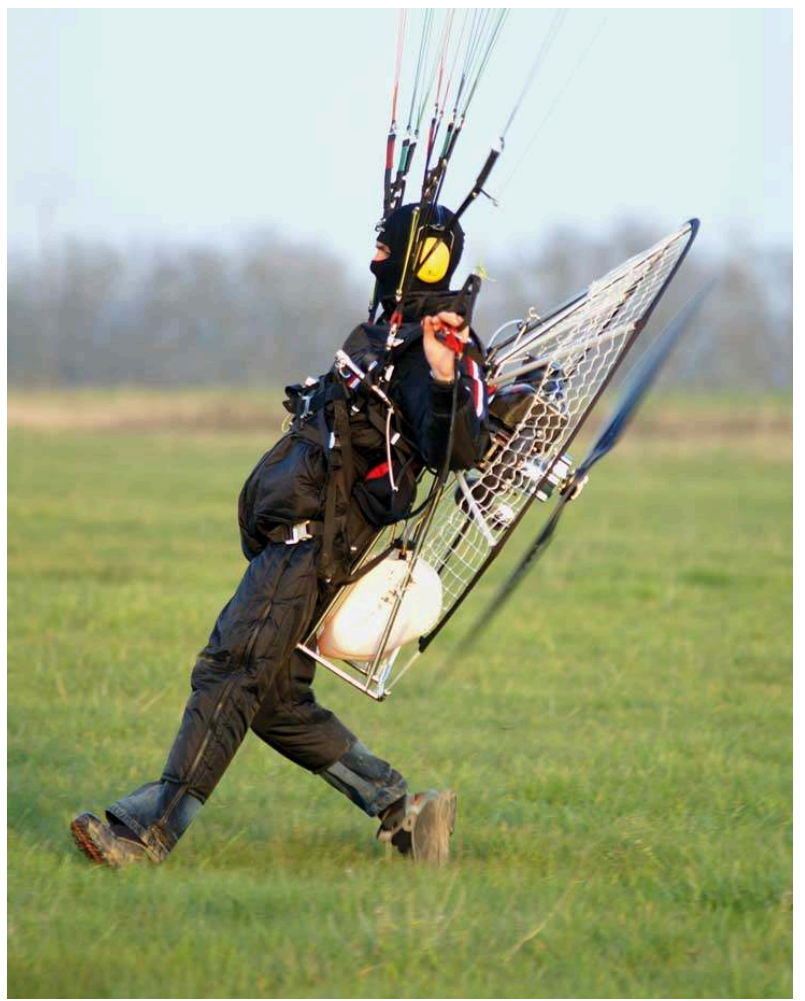

(c) E. Ripoll
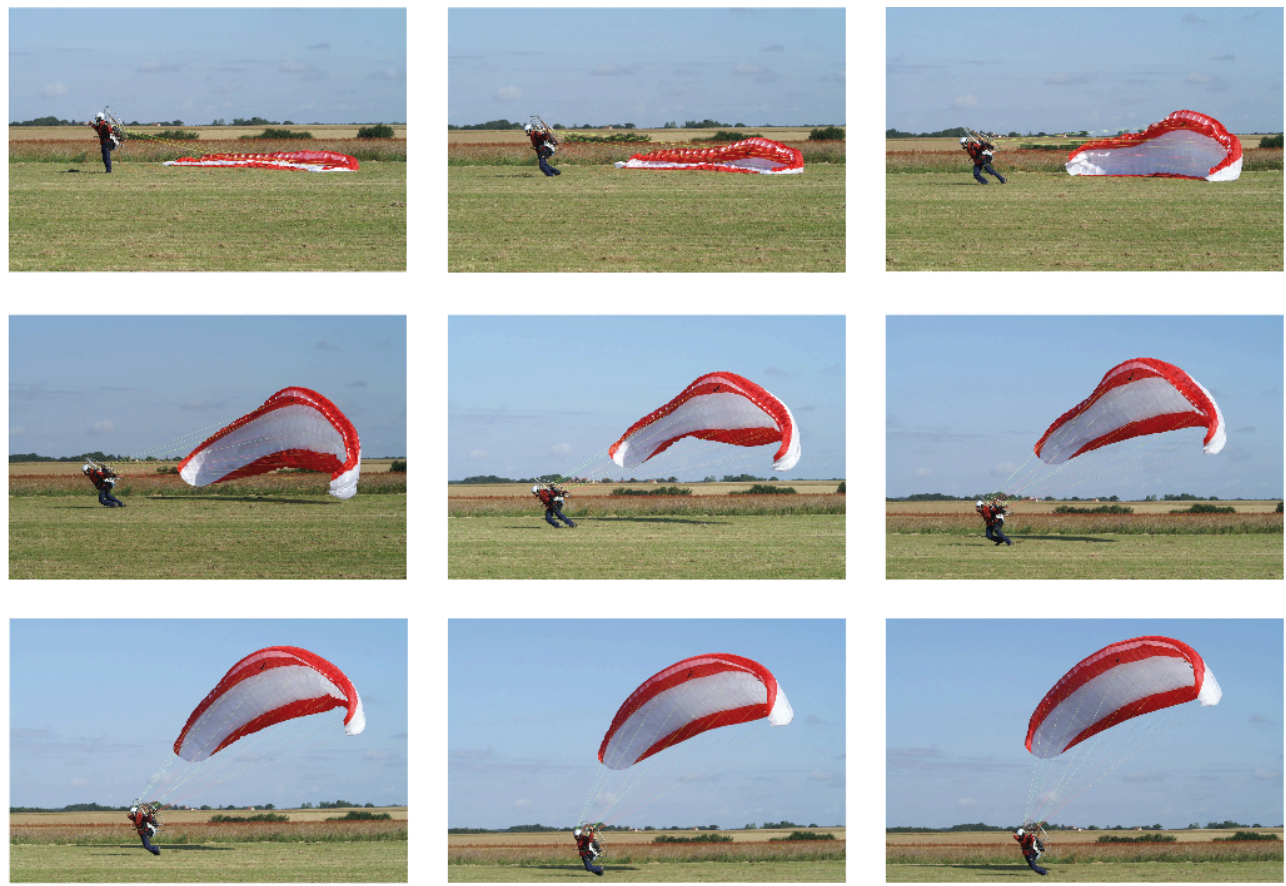

(c) E. Ripoll

La partie délicate à maîtriser est surtout le décollage, qui demande - outre un gros effort physique des gestes adaptés, dans le bon débattement et au bon moment. «En paramoteur t'es pas en l'air par hasard $»$. 


\section{Qu'est-ce que le paramoteur?}

C'est le plus petit des ULM². Le pilote décolle en courant sur quelques mètres, poussé par un moteur dorsal et porté par une aile souple de type parapente. Certains modèles sont biplaces et/ou équipés d'un « chariot ». Un brevet de pilote est nécessaire pour la pratique autonome. La formation ne peut être légalement effectuée que par un instructeur d'ULM, classe paramoteur'. Tant pour les pilotes que pour les instructeurs, un examen national sanctionne la partie théorique commune de la qualification. La pratique et la théorie spécifiques à chaque classe sont évaluées par les instructeurs de pilotes et les centres de formation d'instructeurs. Le brevet est ensuite délivré par l'Aviation civile.

1 La formation initiale des débutants s'effectue auprès d'une population hétérogène du point de vue de l'âge, du sexe, du niveau de pratique, du vécu personnel, des capacités physiques, des styles cognitifs, de la disponibilité temporelle, etc. Les caractéristiques de l'objet matériel dont il est ici question impliquent un important investissement du corps propre (mise en œuvre au décollage, pilotage, atterrissage). Par exemple, le «train d'atterrissage» est constitué des jambes du pilote. Par ailleurs, l'aile est «souple», ce qui demande un "gonflage» de celle-ci par le pratiquant. Ces facteurs rendent la pratique « biplace décollage à pied » très délicate, alors que le chariot - bien plus facile en biplace - permet surtout de former... au chariot.

2 Ces spécificités de l'objet matériel ont des conséquences sur la formation même : la partie délicate à maîtriser est surtout le décollage, qui demande - outre un gros effort physique - des gestes adaptés, effectués dans le bon débattement et au bon moment. Comme le précise un instructeur chevronné d'une catégorie plus classique d'aéronef: «En paramoteur, t'es pas en l'air par hasard! ». L'apprentissage a donc lieu principalement au sol et en groupe. En revanche, dès le premier vol, l'élève est la plupart du temps en solo, sans instructeur à bord.

Les espaces physiques de l'enseignement et des rapports sociaux qui s'y tissent présentent également de grandes disparités: salle de classe, véhicule aménagé, " troquet » mais surtout " pelouse », en lieu et place des usuels club-houses, hangars et pistes du reste de l'aéronautique. 


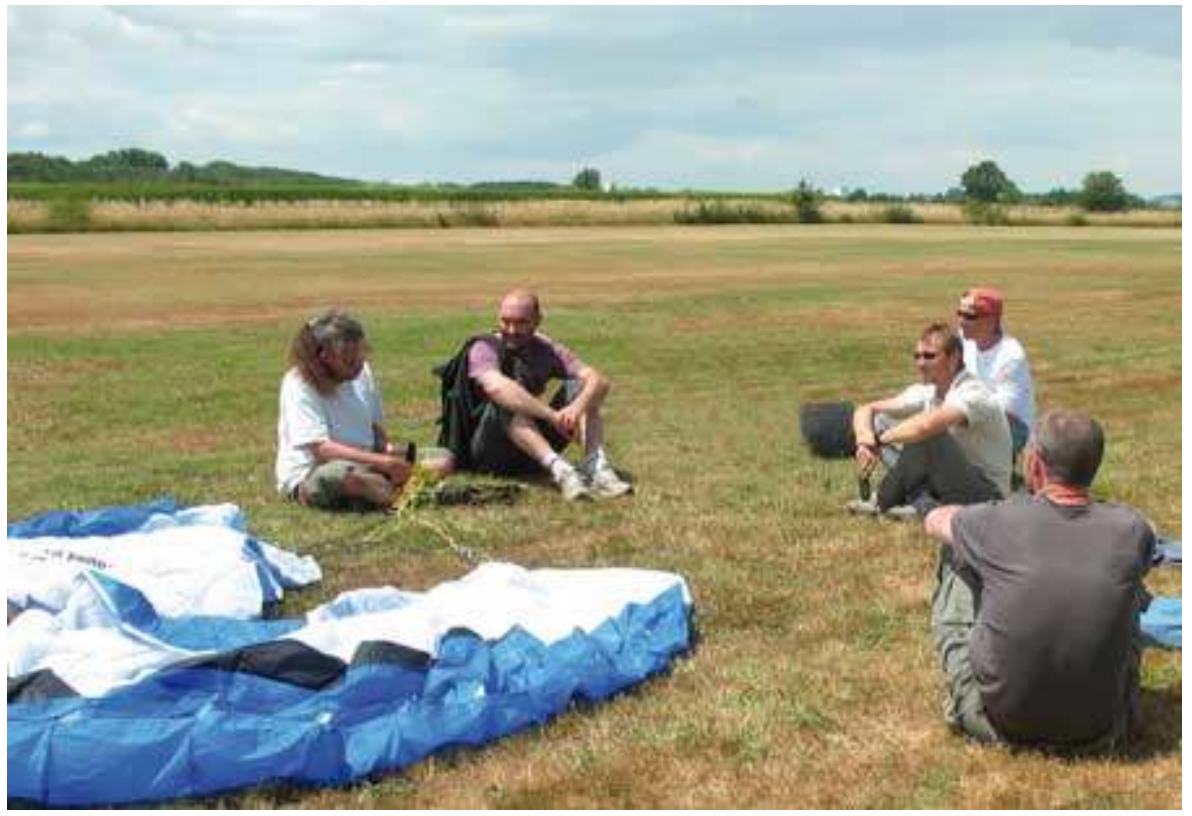

(c) M. Ruet

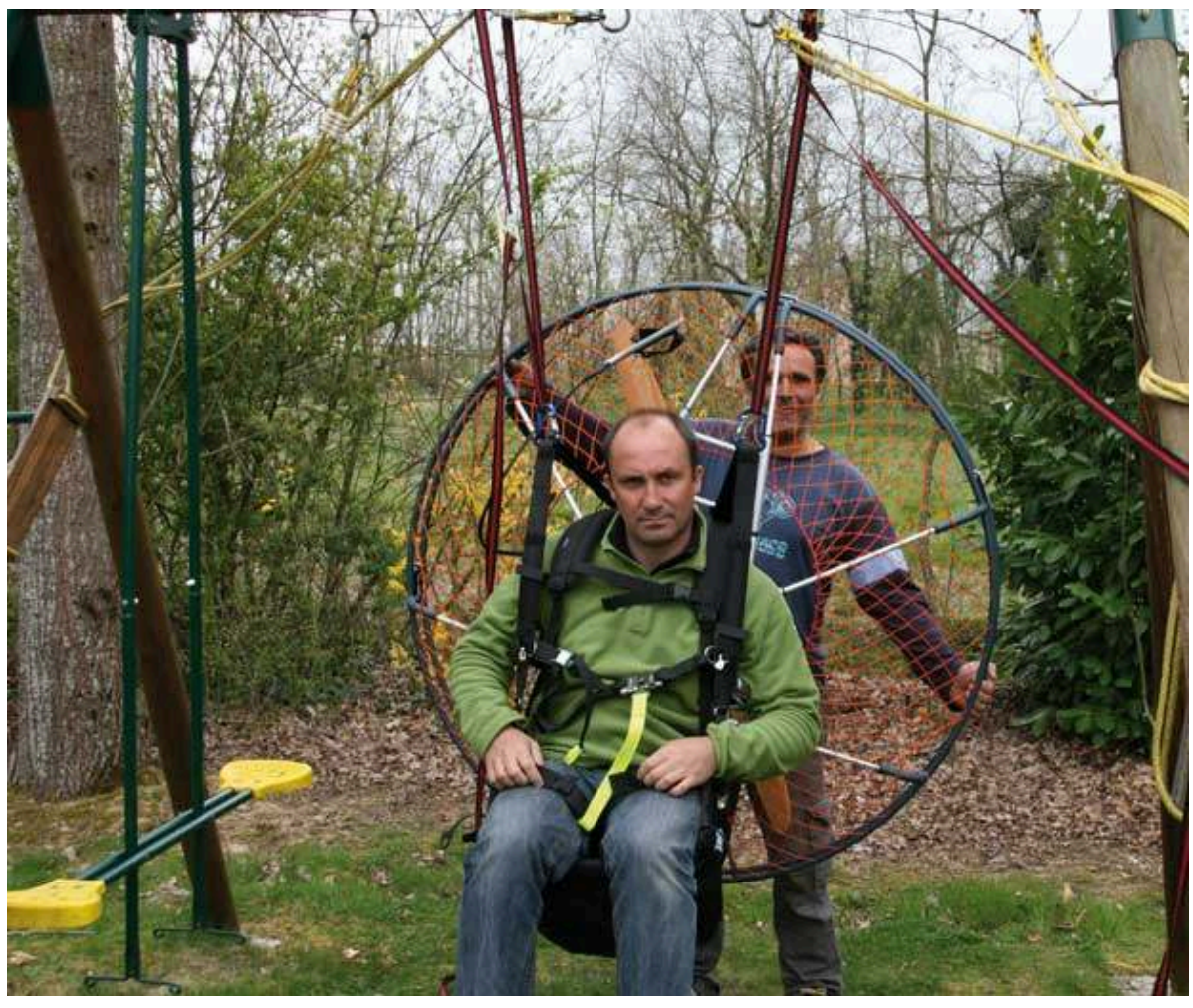

(c) E. Ripoll 


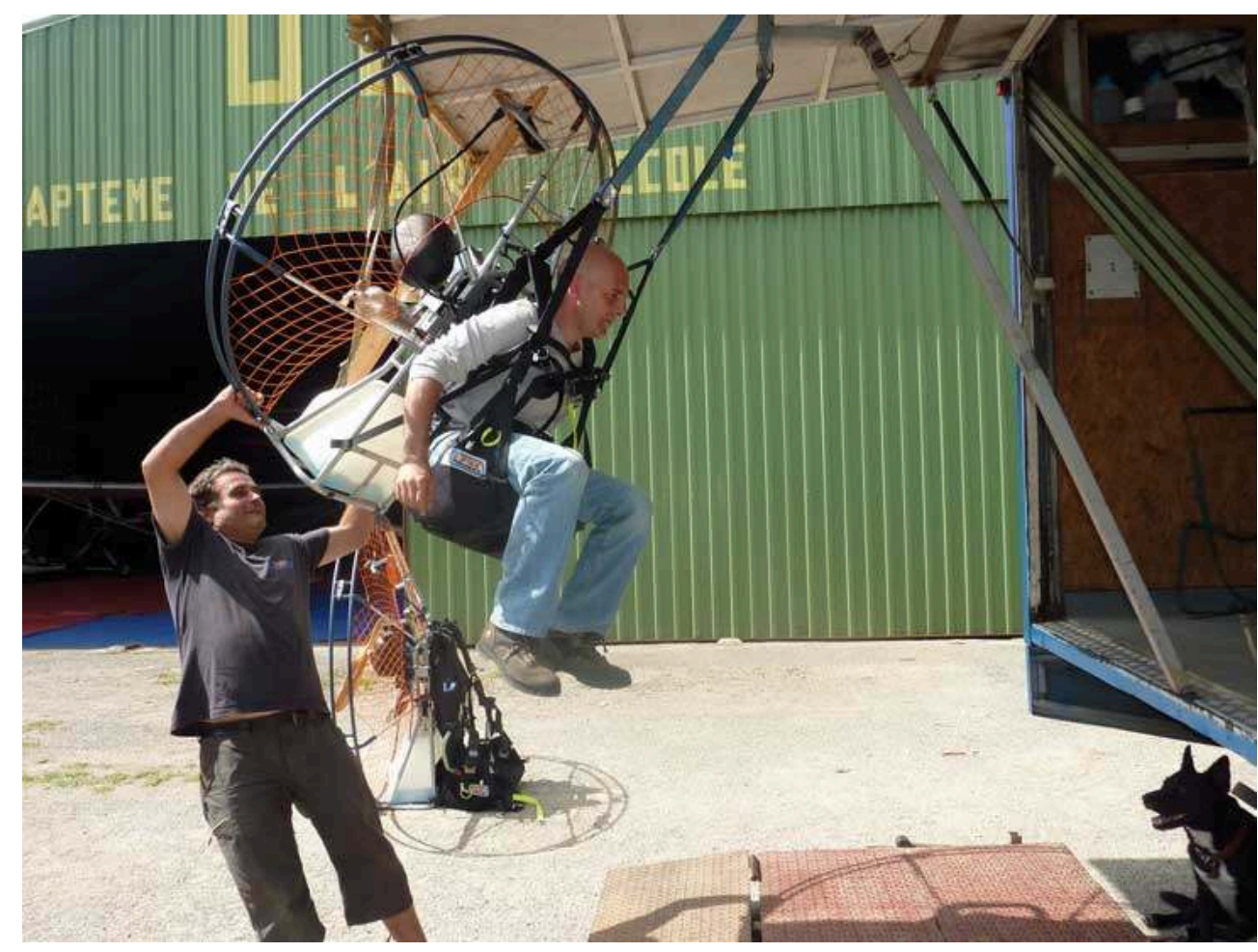

(C) E. Ripoll

Pelouse, portique et balançoire, véhicule aménagé, les espaces sociaux et les supports matériels de la transmission sont variés.

4 L'instructeur paramoteur est un véritable «homme-orchestre »: travailleur indépendant, formateur, vendeur de matériel, mécanicien, gestionnaire de terrain, animateur de groupe, psychothérapeute, etc. Cette multi-activité est fortement liée à l'objet matériel de la pratique. Par exemple, les modèles étant nombreux et très variés, l'acheteur se tournera naturellement vers un matériel qu'il connait, avec lequel il a été formé, auprès d'un instructeur en qui il a confiance.

5 Par ailleurs, les relations interpersonnelles dépassent fréquemment le simple cadre de la formation ou du commerce. La proximité spatiale, l'investissement du corps propre et les périodes non-productives (vent, pluie, aérologie turbulente, etc.) font partie des facteurs qui expliquent l'importance des affects dans la relation instructeur-élève. Il n'est pas rare que des questions d'ordre privé soient abordées. Ce qui fait partie intégrante du travail du formateur : les «facteurs humains » prennent de plus en plus d'importance en aviation ${ }^{4}$.

La quête identitaire, l'implication du corps propre et la hiérarchisation virile (Pénin 2006) étant fortes dans cette activité, le rôle d'animateur de l'instructeur dépasse donc souvent le simple cadre pédagogique. Il subit également des contraintes météorologiques, économiques (concurrence des autres instructeurs, par exemple), psychologiques (stress, horaires), sociologiques. Parmi ces dernières, citons les contraintes administratives, mais aussi la stigmatisation d'une profession à risques aux revenus aléatoires et générant $d u$ bruit, sans oublier la survalorisation du statut «d'aventurier». Physiquement, nerveusement et émotivement, la position charismatique du formateur en paramoteur est exigeante.

7 Test de matériels (parfois délicats), démonstrations en vol, prises de décisions, prises de risques et de responsabilités forment le lot quotidien de l'instructeur. Seul maître à 
bord sur son terrain, il est souvent isolé. Les échanges entre pairs instructeurs, qui sont aussi des concurrents, concernent peu la pédagogie.

8 Les origines sociales et les niveaux de diplôme des instructeurs sont fortement hétérogènes et de grande amplitude. En revanche, la profession est très majoritairement masculine : on compte trois femmes en France, soit environ $2 \%$ des instructeurs en activités. L'âge moyen dépasse quarante ans. L'activité est récente (vingt ans) et en croissance (beaucoup de nouveaux instructeurs); la moyenne d'expérience pédagogique est donc inférieure à dix ans. Peu d'instructeurs viennent de l'enseignement (au sens large), et la formation paramoteur est très courte (aussi bien pour les pilotes que pour leurs formateurs).

9 Les méthodes pédagogiques sont peu théorisées et encore moins algorithmisées ${ }^{6}$. La résistance au savoir livresque est importante et la tendance est encore nettement à la focalisation sur la transmission du savoir-faire incorporé plutôt que sur les cours de navigation, altimétrie ou mécanique de vol. L'élève s'entend parfois dire : «Tiens, voilà un bouquin de théorie, tu viens me voir si tu as des questions ».

10 Si les instructeurs ont des origines variées, il en va de même des objets concrets de la pratique. Les ailes et les moteurs utilisés par les écoles sont nettement plus différenciés que dans d'autres activités aéronautiques. De plus, la rotation des objets matériels est importante : sur un plan diachronique, par l'évolution constante des modèles, l'usure (petits moteurs tournant à haut régime et tissus d'aile très légers) et la progression des pilotes (ou des représentations que ceux-ci s'en font...), mais également par la possibilité et l'usage très courant d'interchanger aile et moteur (deux mousquetons à dévisser). Les pratiques liées à ces objets matériels sont hétérogènes.

11 Les supports pédagogiques présentent également de fortes disparités, en lien avec de multiples variables : la personnalité de l'instructeur, l'appartenance de celui-ci à une communauté de pratiques " paramoteur » (amicale, professionnelle ou commerciale), le choix technique de l'objet matériel, les formes sociales de l'espace, l'utilisation de l'informatique en fonction des compétences, la formation avec des paramoteurs d'un certain type, les cours dispensés dans une salle équipée (tableau, projecteur) ou sur une pelouse, etc.

12 La formation des pilotes (qui sont aussi des acheteurs de matériels) et celle des instructeurs (vendeurs de matériels et prescripteurs de pratiques) font l'objet de "luttes à l'intérieur du champ $»^{7}$, au sens économique et sociologique de ce syntagme ${ }^{8}$. Plusieurs tentatives de mise en place de normes fédérales, voire ISO, ont eu lieu, sans succès jusqu'ici.

Enfin, la compacité du paramoteur, l'inutilité d'un terrain d'aviation pour décoller ou d'un hangar pour stocker, réduisent les espaces sociaux des pratiques adjacentes au vol. Rares sont les écoles ou les clubs qui disposent d'un club-house, lieu de rencontre privilégié dans de nombreuses autres activités aéronautiques (avion, planeur, parachutisme, etc.). Ce manque, ainsi que la concomitance temporelle du développement du paramoteur et de la micro-informatique ont fait d'Internet un espace social conséquent, dans lequel une part du savoir transite en dehors de la transmission du savoir-faire opératoire de l'instructeur. 


\section{La « vicariance » en paramoteur}

En paramoteur, la durée et le contenu des formations, les objets matériels, les techniques du corps, les chaînes opératoires, les pratiques et la transmission du savoir sont ainsi caractérisés par la vicariance, terme polysémique dont je retiens deux acceptions : la variabilité des chaînes opératoires et l'apprentissage dit « apprentissage vicariant $»$.

La variabilité des chaînes opératoires :

«La vicariance des modes opératoires correspond à la possibilité qui est donnée à celui qui réalise une tâche de la réaliser selon différentes façons, sans devoir se conformer entièrement au mode de réalisation prescrit » (Lancry 2009 : 53).

Ce choix de l'acteur paramotoriste présente au moins deux aspects. D’histoire récente, le paramoteur fait l'objet de choix techniques très divers (puissance du groupe motopropulseur, surface et profil de l'aile, type d'accrochage, décollage à pied ou chariot, etc.) et les chaînes opératoires de sa mise en œuvre sont éminemment variées (tant au sol pour la mise en œuvre, qu'en l'air). Ensuite, le «mode de réalisation prescrit » est institutionnellement laissé dans une large mesure à l'appréciation de l'instructeur (en école) et au pratiquant. En paramoteur et en U.L.M. en général, le carnet de vol ${ }^{10}$ n'est pas obligatoire, et une très grande liberté est accordée aux méthodes d'apprentissage et de pratique. Par exemple, le contrôle technique du matériel est très réduit ${ }^{11}$, et il y a peu de contraintes sur la pédagogie elle-même (hormis les limites légales de lieu, de diplôme ou d'assurance).

L'apprentissage vicariant :

«L'apprentissage serait excessivement laborieux, pour ne pas dire hasardeux, si chacun devait s'en remettre exclusivement au résultat de ses propres actions pour savoir quoi faire. Heureusement, de nombreux comportements humains sont appris par observation de modèles : en observant les autres, chacun se fait une idée sur la façon de réaliser de nouveaux comportements, et en d'autres occasions, cette information codifiée sert de guide pour l'action » (Bandura 1977).

Bien avant la remise en cause par Bandura - dans sa théorie sociale cognitive - du behaviorisme fondamentaliste ${ }^{12}$, le système d'essais-erreurs du pilote solitaire, qui cherche à réinventer l'aviation, s'était avéré peu efficace ${ }^{13}$. L'apprentissage vicariant par observation de "l'autre » au sens large - permet une gestion optimum du temps et des risques de l'apprentissage.

En paramoteur, le copiage n'est pas stigmatisé comme dans une formation scolaire. Bien au contraire, un des objectifs de l'instructeur est de gagner du temps (principalement pour des raisons économiques ${ }^{14}$ ), en encourageant les échanges et en diffusant le savoir non seulement auprès de chaque individu, mais dans le groupe de ses élèves, et si possible au-delà. Dans cette démarche, il se trouve souvent en conflit avec d'autres instructeurs ainsi qu'avec des entités professionnelles, associatives ou virtuelles (forums Internet en particulier) aux motivations et objectifs divergents.

Dans cette lutte pour la transmission, le formateur en paramoteur dispose cependant d'un atout important: l'école est un lieu privilégié d'aperception sociologique et d'adhésion aux valeurs d'un groupe sur lequel il peut exercer une domination. Celle-ci souvent charismatique ${ }^{15}$ - permet d'établir des relations d'intimité et de respect, dans le cadre desquelles l'imitation des comportements d'autrui (apprentissage vicariant) est rendue possible et prend toute sa dimension de vecteur culturel. 


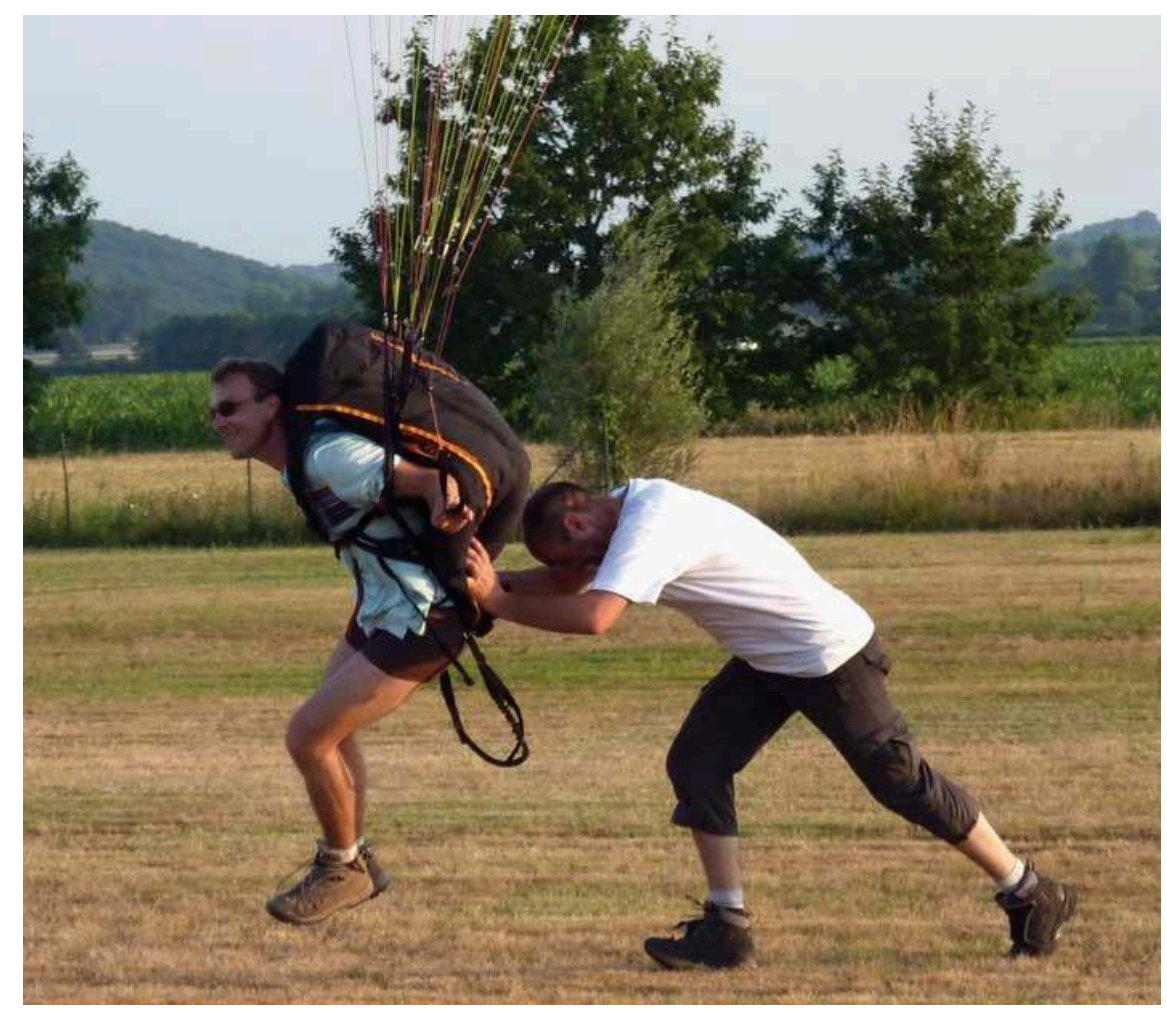

(c) E. Ripoll

Les exercices au sol impliquent le corps, préparent à l'efficacité de l'action sur la matière et permettent l'apprentissage vicariant.

\section{De la chaîne opératoire à la méta-chaîne opératoire}

Ma première préoccupation, lors de ma recherche sur la transmission du savoir en paramoteur, fut de décrire finement les objets et les techniques du corps, puis de tenter d'observer et de décrire les chaînes opératoires.

«Pour qui prend au sérieux la proposition générale de Mauss (1968) d'élargir le champ de l'ethnologie à ces humbles et triviaux comportements humains que sont les techniques du corps, et, a fortiori à toute action technique, comprendre en quoi une opération matérielle est propre à un groupe particulier, c'est d'abord tenter de déchiffrer la manière dont divers éléments (énergies, outils, gestes, connaissances, acteurs, matériaux) sont mis en relation au cours de processus qui modifient un système matériel : disons la fabrication d'une poterie, la mise en vol d'un aéroplane ou la préparation du café du matin [...] » (Lemonnier 2004).

Mais aussi: "se pencher sur les dimensions les plus matérielles des objets, comprendre leur fabrication ou leur fonctionnement permet surtout de mettre au jour des pans entiers de systèmes de pensée ou de logiques sociales qu'on ne saurait repérer et comprendre autrement » (Ibid.).

J'ai pourtant été confronté à une limite, lors de l'étude de ces «dimensions les plus matérielles des objets", en particulier dans les phases de formation. Impliqué scientifiquement sur mon terrain depuis 2005 - mais professionnellement depuis vingt ans - je repérais dans la transmission du savoir, des chaînes opératoires superfétatoires, fugaces, camouflées, versatiles... qu'un technologue culturel non impliqué16 aurait eu bien du mal à saisir, ou au moins à expliquer. 


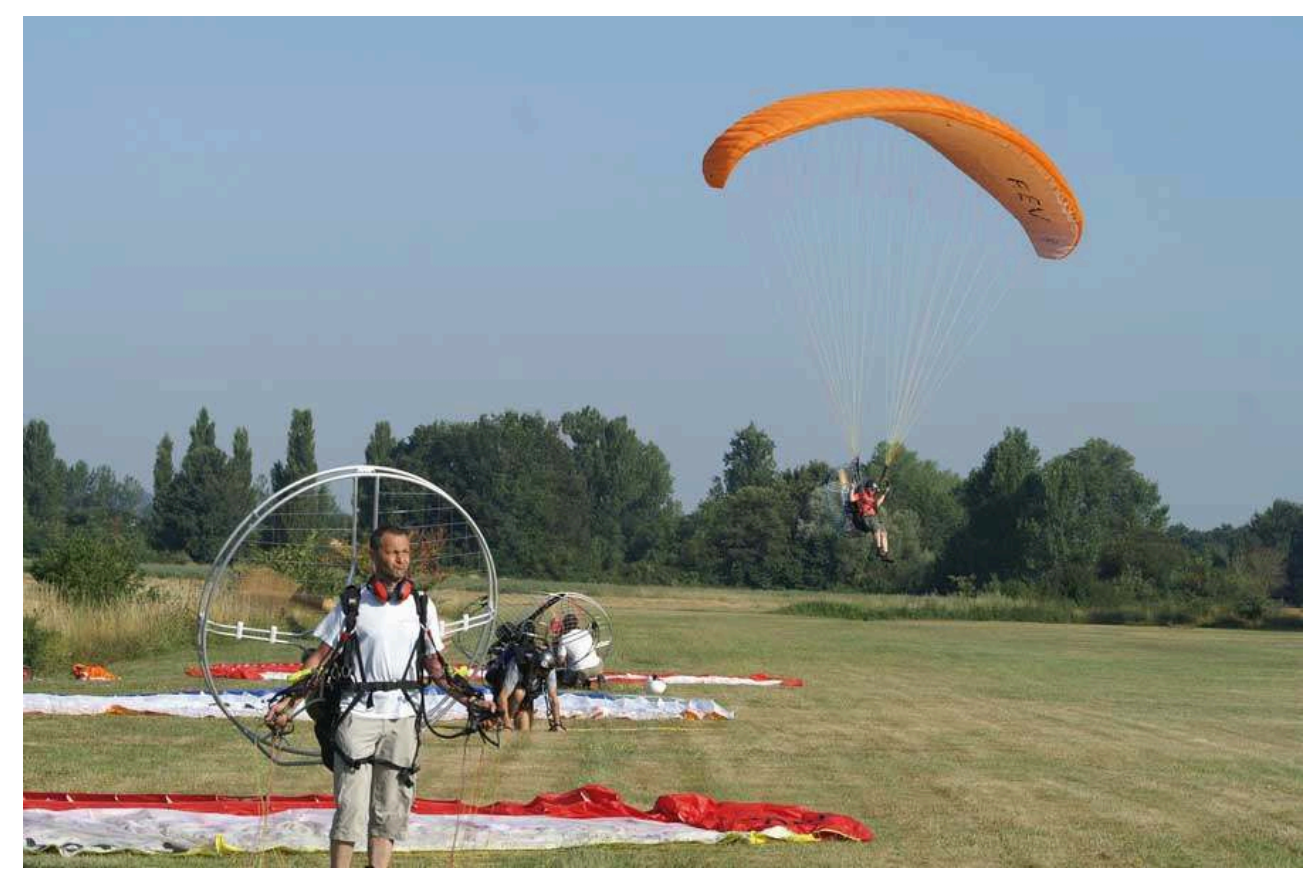

(C) E. Ripoll

Les paramotoristes « essaient » souvent les ailes et les moteurs des autres pratiquants.

21 Ma réflexion a porté en particulier sur la mécanique de vol du paramoteur; sur les rapports entre l'économique et le technique (en paramoteur, le pédagogue est aussi un commerçant), mais également sur un questionnement épistémologique du rapport altérité-implication ${ }^{17}$ du chercheur (qui est un point secondaire de cet article mais que je souhaite rappeler). Enfin, j'ai été amené à ressentir la nécessité d'un outil permettant d'affiner cette « chaîne opératoire [qui] constitue la matière première de l'ethnologie des techniques "(Ibid.), en particulier dans les situations de transmission de savoir.

J'appelle " méta-chaîne opératoire ", la démarche physique et mentale qu'effectue - dans et hors l'action - celui dont le but est de transmettre un savoir, avec une intentionnalité qui va au-delà de l'action sur la matière. En d'autres termes, il s'agit de prendre en compte - lors de la description d'une activité technique - la réflexivité, l'acceptation et la gestion des risques par l'instructeur, pour lui-même et pour l'autre, avec un investissement affectif.

Dans cette méta-chaîne opératoire, l'instructeur gère l'entourage physique et humain, et la condition physique et psychique de l'élève. Il doit faire effectuer l'exercice dans un créneau météorologique compatible avec l'activité prévue, avec le matériel adapté, etc. Il doit également choisir un créneau horaire compatible avec la chronopsychologie humaine (essayez donc de faire un cours de réglementation après le repas de midi, c'est la sieste assurée!). Enfin, il doit positionner l'exercice sur un plan diachronique, en s'assurant que l'élève a intégré les pré-requis nécessaires à l'activité en cours. En effet, la transmission du savoir concernant une action sur la matière exige fréquemment la maitrise d'une action précédente. Par exemple, le décollage nécessite une certaine compétence en gonflage de l'aile. 


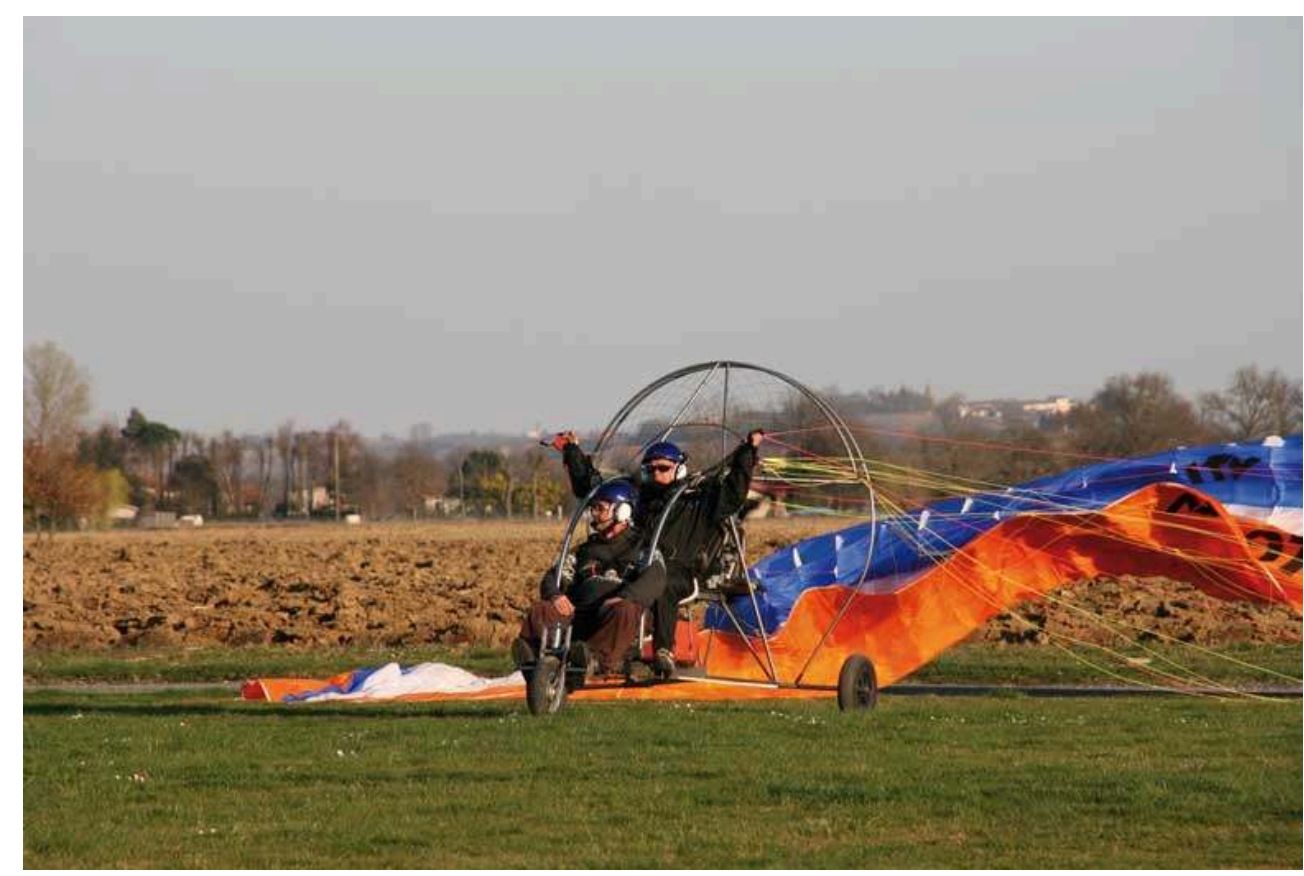

(c) M. Ruet

La pratique du biplace reste une exception. L'enseignement au sol a lieu en groupe, alors que l'élève est le plus souvent seul à bord dès le premier vol.

Le formateur porte donc son regard de l'extérieur de l'action et de l'intérieur de l'action; il prend en compte des humains, des objets matériels et un environnement physique et social; il fait des choix temporels et matériels, etc. Tout ceci place l'instructeur paramoteur dans une situation autrement plus complexe que la « simple» application d'une chaîne opératoire concernant les seules techniques directement liées aux phases du vol. C'est d'autant plus vrai dans le cas de méta-chaînes opératoires "à long terme ", quand la motivation de transmettre des représentations dépasse l'objectif à court terme d'une action sur la matière.

L'intentionnalité du formateur peut donc aller jusqu'à une volonté consciente d'enculturation de l'élève. Toutefois, son habitus personnel impose également une transmission culturelle inconsciente. Prenons l'exemple de la transmission (primordiale en aviation) des représentations de «ce qui est possible»: nous savons que les représentations familiales ont une influence déterminante sur les aspirations des étudiants en ce qui concerne les diplômes ${ }^{18}$. Il en va exactement de même pour la formation des élèves en paramoteur. Les représentations de l'instructeur l'amèneront à effectuer des méta-chaînes opératoires inconscientes qui détermineront des comportements chez ses élèves. Par exemple, voler en conditions orageuses devant ses élèves, les conduiront à envisager que c'est possible.

Dans l'action, la chaîne opératoire de l'instructeur peut être homothétique ${ }^{19}$ de celle demandée à l'élève : par exemple, j'incite les instructeurs à effectuer dans leur école exactement les mêmes types de vols que ceux qu'ils demandent à leurs élèves. Nous sommes alors dans la monstration. Ce niveau de transmission du savoir est couramment observé dans les pratiques ne disposant pas de «formateurs »: jeux de billes, sexualité, cuisine familiale, etc., mais aussi dans les débuts de pratiques aéronautiques comme le parapente ou le paramoteur. 


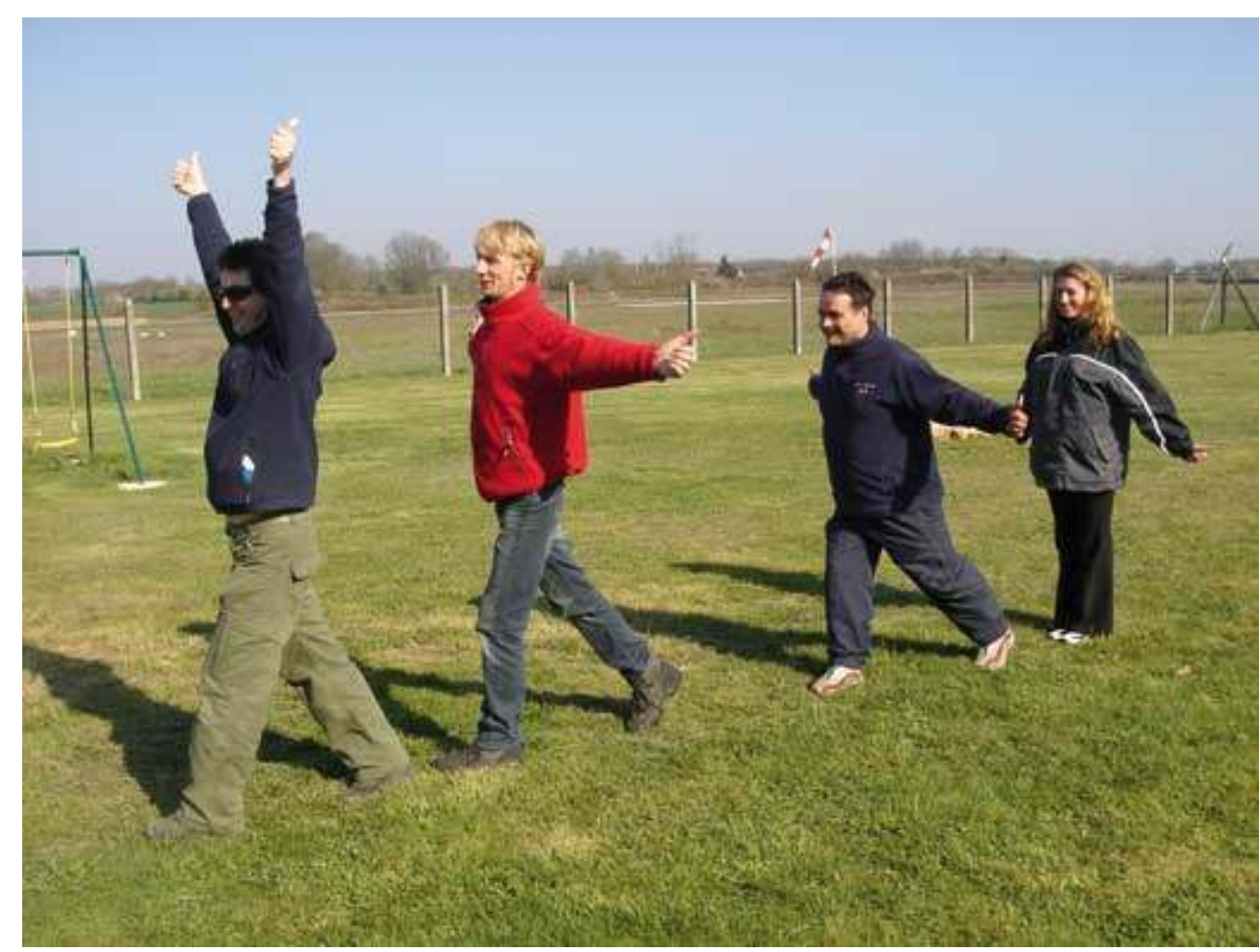

(C) E. Ripoll

L'école en paramoteur est un lieu privilégié d'aperception sociologique et d'adhésion aux valeurs d'un groupe.

27 Avec l'institutionnalisation (création du brevet de pilote et de la qualification d'instructeur), un deuxième niveau apparaît. L'élève-instructeur apprend alors, d'une part, à transmettre un savoir " algorithmisé », c'est-à-dire déposé concrètement sur des supports physiques: mode d'emploi, aide-mémoire, manuel, check-list, ouvrage de référence, etc. D'autre part, le stagiaire acquiert des techniques pédagogiques concernant l'action sur la matière et l'acquisition de savoir-faire incorporés. L'élèveinstructeur va ainsi apprendre à montrer en décomposant et en exagérant, à expliquer, à répéter, à faire effectuer, à renforcer positivement, à corriger, à recommencer en variant les approches et les méthodes, à adapter continuellement le fond et la forme de son discours aux élèves présents et au contexte, à mettre en relation les savoir-faire « algorithmisés » et incorporés.

Le troisième niveau - celui que je propose d'appeler la méta-chaîne opératoire - est peu discernable à première vue de la méthode « explication / monstration / exécution par l'apprenti / correction ». Mon objectif est de démontrer qu'elle est pourtant un facteur d'enculturation déterminant, en particulier dans la gestion ultérieure du risque par les acteurs, en relation directe avec les techniques, les pratiques et les représentations transmises par, pour et à travers l'objet matériel. 


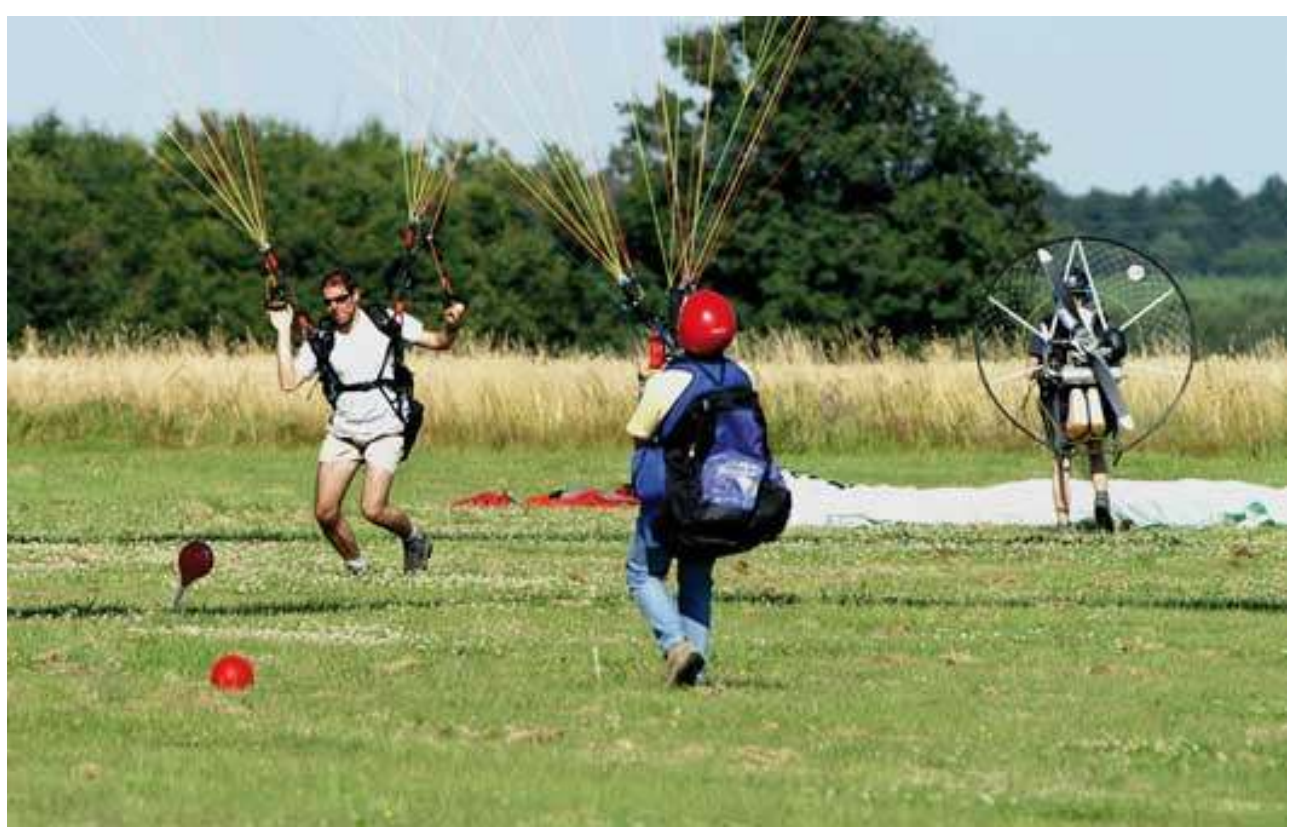

(c) E. Ripoll

L'apprentissage vicariant - par observation de « l'autre » au sens large - permet une gestion optimale du temps et des risques de l'apprentissage.

\section{Une méta-chaîne opératoire de l'instructeur}

Imaginons un instructeur-revendeur faisant effectuer une tentative de décollage à un élève, avec une aile de modèle A. Nous sommes en présence d'une action sur la matière dont la finalité semble univoque: l'élève a déjà fait plusieurs vols, mais le désir d'acheter du matériel n'a pas été exprimé; le discours des deux interactants, leurs pratiques et une part de leurs représentations positionnent l'exercice dans une relation de transmission de savoir. Pourtant, la motivation des deux parties est tacitement « mixte ». L'élève est très près d'obtenir son brevet, et il est averti de l'impossibilité de louer du matériel. Il est donc "mûr » pour investir, et l'instructeur le sait. L'efficacité de cette action sur la matière, totalement identique à celle des précédents vols, comporte donc une dimension économique. Une longue observation participante permet de discerner cette subtilité liée à la chronologie de la formation. En revanche, il faut un œil extrêmement exercé et une expérience individuelle longue du matériel et de l'enseignement pour repérer une méta-chaîne opératoire. En effet, le réglage de l'aile (positionnement des trims ${ }^{20}$ ) n'est pas tout à fait optimum; le « top gonflage ${ }^{21}$ est donné par l'instructeur une fraction de seconde après le petit souffle de vent idéal. La tentative échoue. Plusieurs heures plus $\operatorname{tard}^{22}$, un autre essai est effectué avec une autre aile, de modèle B. Cette fois, les données matérielles et immatérielles sont subtilement différentes : réglage parfait, choix du meilleur créneau et empathie plus enthousiaste de l'instructeur. L'élève réussit son gonflage et l'instructeur face à lui donne l'autorisation de décollage (par radio et/ou en faisant tourner son bras au-dessus de sa tête). Dans le soleil couchant, l'élève effectue un vol magnifique et lors du posé, il reçoit l'accolade de son maître: "Je crois que ce soir tu paies le coup». Ce qui veut dire que le brevet va être signé. Que croyez-vous qu'il advienne ? C'est cette dernière aile qui est vendue dans la foulée..., or c'est également sur celle-ci que l'instructeur réalise sa meilleure marge commerciale. La méta-chaîne opératoire peut s'arrêter là. Il n'est 
d'ailleurs parfois même pas nécessaire de la mettre en action : certains instructeursrevendeurs font acheter l'aile avant la formation. Dans d'autres écoles, celui qui n'a pas encore acheté « attend son tour » beaucoup plus que les autres (et finit par comprendre qu'il doit investir). À l'inverse, certains instructeurs sont de si piètres revendeurs qu'ils se font «souffler » leurs clients par des collègues. Jusqu'ici, rien de spécifique à cette pratique. La méta-chaîne opératoire va au-delà de la chaîne opératoire dans la mesure où elle correspond tout à la fois à une intégration des savoirs et des pratiques et à une mise à distance réflexive et affective. Mais qu'en est-il de la transmission culturelle ?

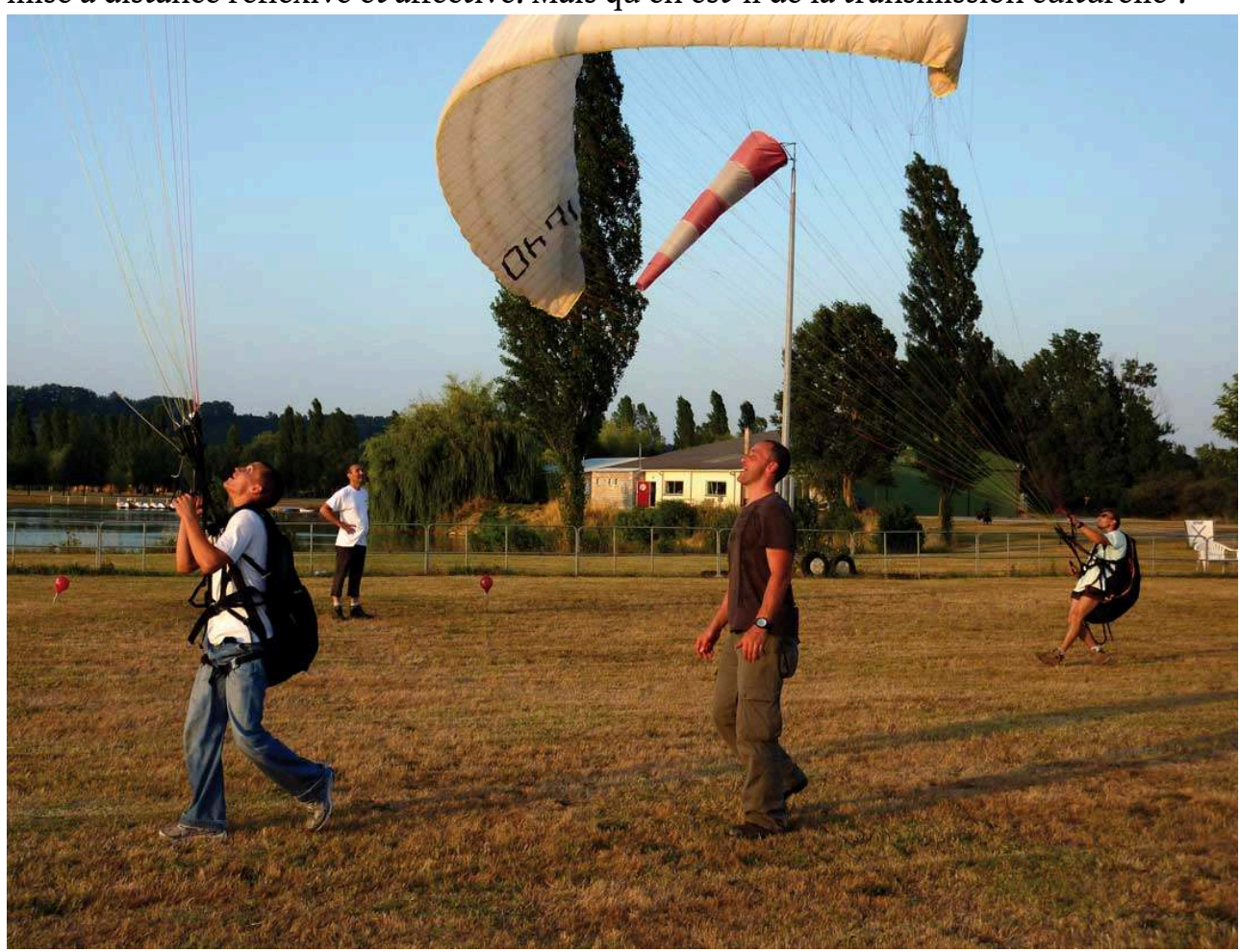

(c) E. Ripoll

Démarche physique et mentale dans et hors l'action, la méta-chaîne opératoire est plus difficile à décrire que les chaînes opératoires.

\section{Une « tâche stratégique » ${ }^{23}$ de la transmission culturelle}

Une connaissance approfondie de l'objet matériel va amener le chercheur à affiner sa première conclusion strictement limitée à l'aspect économique. En effet, l'instructeur observé dans le cadre de mon étude effectue un choix technique des moteurs associés aux ailes. Les caractéristiques de la première aile essayée (de type A) justifieraient le fait de proposer une motorisation plus puissante, laquelle offrirait une meilleure marge commerciale. Il y a donc dans les deux cas, une contradiction des intérêts économiques entre le choix technique de l'aile et celui du moteur. Le chercheur doit faire preuve de patience et chercher des résurgences de la première méta-chaîne opératoire observée.

Lors d'un cours théorique sur les profils, il peut constater que l'instructeur explique clairement la nécessité d'un réglage plus précis des trims sur le type de profil du modèle A par rapport à celui du modèle $B$. Cette contrainte pour réussir un gonflage de l'aile par vent faible est compensée par la plus grande capacité du profil du modèle A en 
situation de vent fort (et donc en aérologie plus turbulente). Sommes-nous alors en présence d'une autre étape de la " manipulation ", visant en fin de compte à convaincre les élèves (futurs acheteurs), de porter leur choix sur l'aile la plus facile à régler (et fournissant la meilleure marge commerciale) ? Pas forcément, car un autre cours sur les effets-moteur ${ }^{24}$ montre que l'instructeur-revendeur incite clairement ses élèvesclients à s'orienter vers des moteurs modérément puissants, adaptés aux ailes de type $B$, et moins chers. La confrontation de plusieurs méta-chaînes opératoires de cet instructeur, à différents moments, met donc au jour un rapport direct entre le choix technique (modèle $\mathrm{B}$ et moteur modérément puissant) et un objectif à plus long terme : inciter ses pilotes à préférer les conditions de vent faible. Dans ce cas, aux dimensions «apprentissage du vol » et « vente de matériel » s'ajoutent d'autres considérations ou intentionnalités relatives à la sécurité.

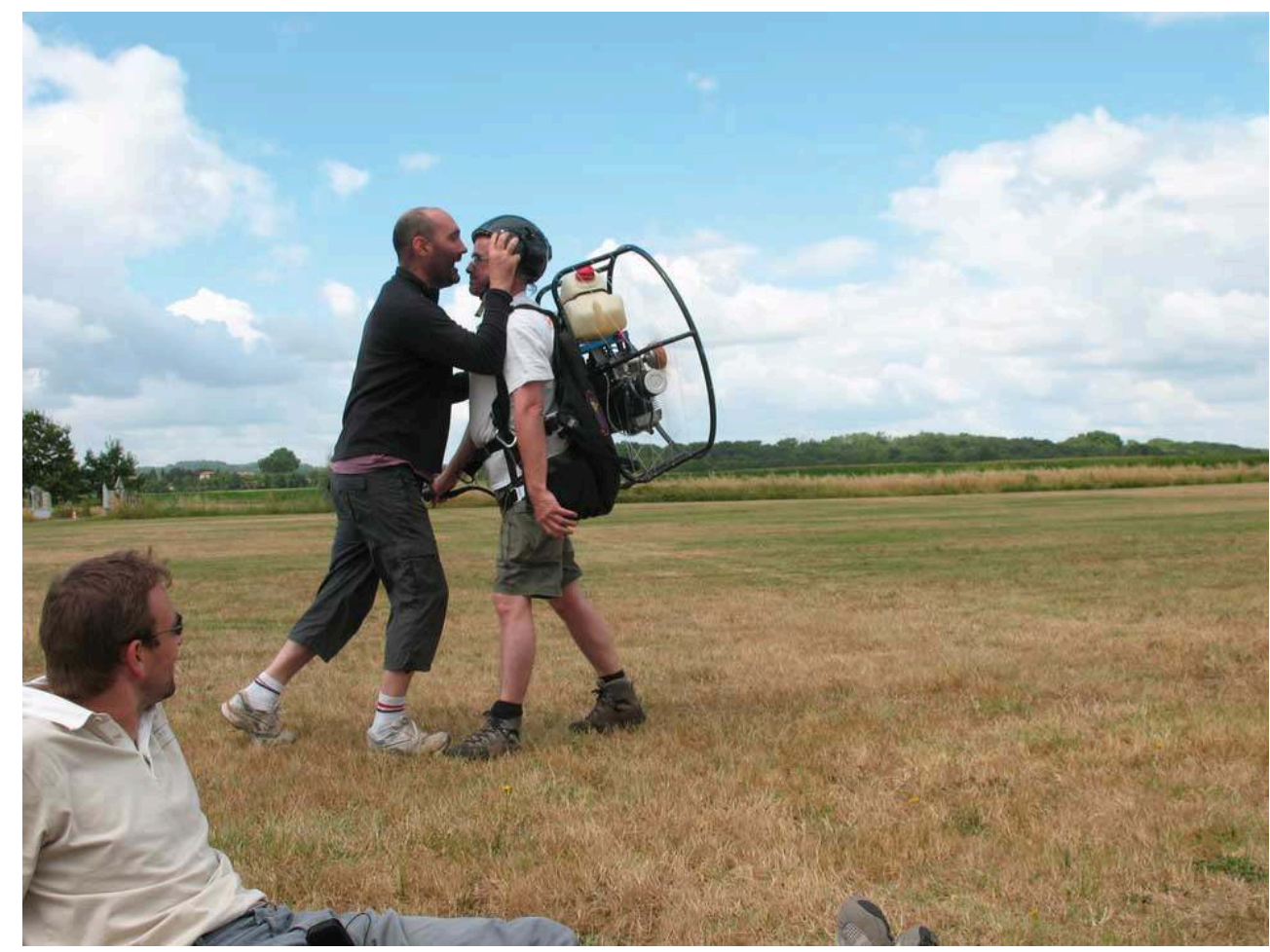

(c) E. Ripoll

Proximité corporelle, lors d'un exercice délicat de poussée moteur. L'instructeur enseigne l'efficacité d'une action sur la matière, mais transmet également des comportements et une culture de la sécurité, " par, pour et au travers de l'objet matériel ». Nous sommes même, dans ce cas précis, à un niveau « au-dessus » : cette image montre une transmission de méta-chaîne opératoire, l'un des intervenants étant instructeur stagiaire et l'autre, son formateur.

Les entretiens (à condition de poser les bonnes questions) et les comparaisons entre écoles permettent également de moduler une vision strictement économique de la méta-chaîne opératoire étudiée. L'instructeur observé déclare :

«Oui, je gagne plus sur chaque modèle B vendu, mais si je vendais des modèles A, je perdrais moins de temps à expliquer le gonflage : avec du vent, tout le monde $\mathrm{y}$ arrive, et ils croient qu'ils vont être de meilleurs pilotes en volant par vent fort, alors qu'en apprenant à gonfler correctement et à voler par vent faible, ils seraient plus en sécurité ! ».

31 Nous avons donc plusieurs cultures de vol ${ }^{25}$, de vente et d'enseignement, dans lesquelles les phénomènes de mode et les motivations des "prescripteurs » (en 
particulier les instructeurs et les intervenants des forums Internet) s'opposent et déterminent des choix techniques.

En aval ${ }^{26}$ de ces choix techniques, les fabricants d'ailes conçoivent, commercialisent et établissent leur communication sur des modèles «demandés "; alors qu'en amont, la demande est partiellement le résultat de méta-chaînes opératoires des instructeurs. Au bout du compte, quand la meilleure marge commerciale apparait sur un produit, elle est la conséquence de luttes sociales qui déterminent une hiérarchisation des fabricants. Celle-ci est bien sûr le résultat de la concurrence entre constructeurs, mais également entre instructeurs qui vendent des produits différents. S'y rajoute l'influence des forums Internet. Sur ces derniers interviennent des pratiquants de niveaux très variables, ainsi que des pilotes plus ou moins impliqués avec des professionnels ; mais aussi parfois des instructeurs, et plus rarement des fabricants.

Les constructeurs qui ont le vent en poupe disposent d'un meilleur chiffre d'affaires, et peuvent ainsi proposer de meilleures conditions aux instructeurs-revendeursprescripteurs. En effet, cette hiérarchisation a pour conséquence un volume de vente plus important pour les leaders, permettant en retour de meilleures marges commerciales et des investissements (utilisés pour les homologations, la conception, la publicité, etc.) qui participent à leur tour au volume de vente.

Cependant, la situation de leadership est fragile. Dans un marché restreint, où les relations interpersonnelles jouent un rôle décisif, l'entretien d'une position dominante par un fabricant fait appel à des choix qui touchent autant le social que le technique : publicité ou contacts personnels; influence sur les formations ou prise en compte de l'avis des instructeurs; image de marque "père tranquille " ou "aventurier »; interventions dans les forums, sur les salons ou dans les écoles ; adhésion à la "mode " de voler vite par vent fort, ou promotion ${ }^{27}$ de pratiques de vol plus «traditionnelles »; enfin et surtout, les fabricants de matériel sont continuellement dans l'interrogation: «tendance ou fait » (Leroi-Gourhan 1943 : 27-35). En effet, la demande de la clientèle semble parfois s'orienter vers une catégorie de produits (surface et/ou profil d'aile, puissance ou type du moteur, etc.), mais sommes-nous alors devant un phénomène durable (tendance) ou plus ponctuel (fait)? La faible profondeur temporelle de cette pratique ne nous permet guère de trancher cette question aujourd'hui.

Les fabricants dominés ou les outsiders n'ont d'autres choix que d'appliquer des "stratégies de subversion", en prônant une autre «culture » (par exemple, le vol par vent fort et les ailes de type A). Des stratégies du même ordre peuvent être décelées dans des chaînes opératoires d'instructeurs et peuvent permettre d'établir une typologie de styles d'enseignement. Sans entrer dans les détails, je retiendrai deux idéaux-types au sens wébérien:

$\mathrm{X}$ : «Un instructeur doit montrer à l'élève ce qu'il doit faire, tout ce qu'il doit faire,

et rien que ce qu'il doit faire. »

$\mathrm{Y}:$ «Je montre à l'élève tout ce qu'il ne faut pas faire et que moi je sais faire ».

36 En dehors des jugements de valeur et de la hiérarchisation virile (Pénin 2006) (X considérant $Y$ comme un "bras cassé ", et $Y$ jugeant $X$ comme un " vole petit »), chacun transmet des normes (ou des transgressions de normes), des valeurs et des prescriptions (en particulier de choix techniques).

Les institutions au sens large ne sont pas étrangères aux conflits internes qui sillonnent la pratique : l'école est le moment clé de l'enculturation, où des " tâches stratégiques " effectuées par des instructeurs indépendants difficiles à gérer vont déterminer des flux 
d'adhérents, du chiffre d'affaires et des comportements. Fédération, fabricants et Aviation civile sont donc fortement désireux d'être parties prenantes de la formation des pilotes et des instructeurs, lesquels adhèrent, luttent ou ignorent, selon des convictions qui furent partiellement forgées au cours de leur propre formation.

Les enquêtes existantes - ou les institutions qui tentent de gérer le mouvement ULM et le paramoteur en particulier - présentent une caractéristique commune : la dimension matérielle de l'objet et l'importance culturelle de la transmission du savoir ne sont guère prises en compte. Par ailleurs, les intervenants ne sont souvent des spécialistes ni de l'enseignement ni de la technologie culturelle... ni même parfois du paramoteur.

Pourtant, le pratiquant a parfois une compréhension étonnamment claire des métachaînes opératoires qu'applique l'instructeur. Ce qui devrait inciter certains « spécialistes » à plus de modestie. L'exemple suivant illustre cette compétence et, dans le même temps, une méta-chaîne opératoire dans un autre domaine :

"C'était une de mes toutes premières interventions de pompier volontaire: "Départ FPT, feu d'établissement industriel"... me retrouver en binôme avec un ancien d'une telle importance, en devient presque un honneur... dans le fourgon, il prend ses gants et se les met sur la tête et commence à aboyer... j'éclate de rire. Mais comment un homme qui possède un tel vécu, peut-il partir en intervention... et faire "le cocker" ? Et il continue. Il m'attrape par le ceinturon de sécurité, me tire un grand coup et je tombe à terre la tête sur ses genoux, et là c'est parti, il me chatouille sans plus s'arrêter, j'en peux plus, les larmes aux yeux j'arrive à reprendre mon souffle et le regarde tirer la langue comme un chien qui bronze au soleil. Je ne trouve pas ça très sérieux, un mythe commence à dégringoler. "Regarde j'habite la maison qu'on voit au loin”. Et comme un imbécile j'exécute les ordres, je pose mon regard sur le paysage qui défile à toute allure, j'ai à peine le temps de comprendre qu'il n'y a aucune maison à l'horizon, je me retrouve la gueule contre le carreau. D'un air super sérieux il me dit: “T'avais qu'à mettre ton casque !" Pendant que j'étais sur ses genoux, il a eu le temps de voir si j'avais ma cagoule. Comment je le sais? Parce que je me souviens l'avoir mise dans le mauvais sens en entrant dans l'engin. Il m'a tiré sur le ceinturon, pour savoir s'il était bien accroché comme il faut, et m'a mis la tronche sur le carreau, maintenant, j'ai toujours le casque quand je monte dans l'engin. Ce jour-là, je suis parti dans la précipitation, mais lui était sûr que mon équipement était bon. L'intervention s'est très bien passée, mais maintenant, je sais qu'avant de mettre le pied à terre, avant même de penser à le faire, avant même de réfléchir à ce que je vais faire ce week-end, je dois savoir si je suis prêt $»^{28}$. 


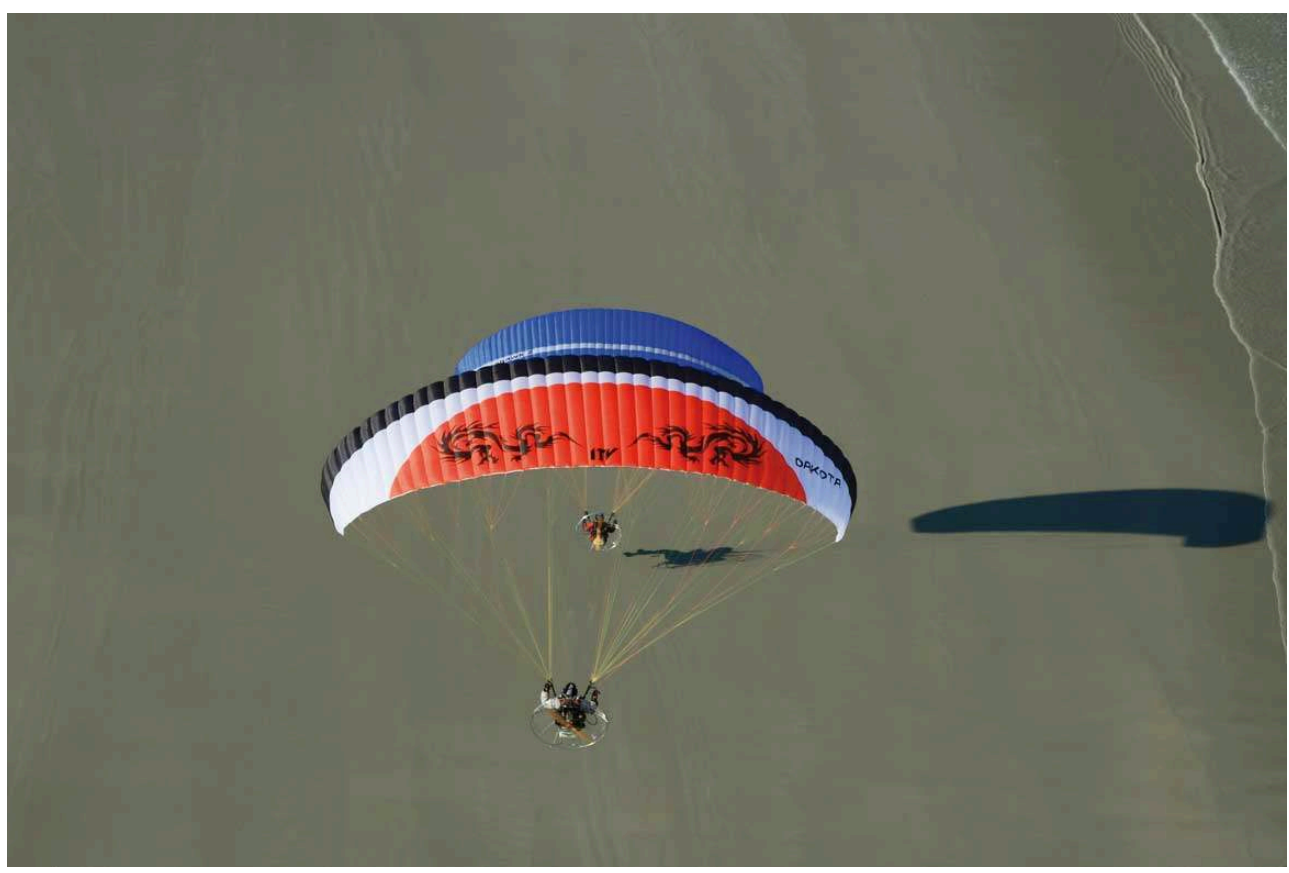

(c) D. Dréano

Le vol est un moment magique... qui se mérite. Voler en sécurité est la conséquence d'une transmission de savoir et de culture.

\section{Caractéristiques de la méta-chaîne opératoire}

Dans l'état actuel de ma recherche, j'ai relevé sur mon terrain d'étude les propriétés suivantes d'une méta-chaîne opératoire :

Elle est souvent fugace (ce peut être un simple regard, ou une action extrêmement brève), difficile à observer sans une très longue implication et une connaissance fine du terrain, des objets matériels et des chaînes opératoires.

Elle est sujette à des résurgences dans le temps, tout aussi fugaces et versatiles. Par exemple, un cours sur la motorisation - plusieurs jours après - peut subtilement appuyer une méta-chaîne opératoire qui concernait une aile.

Elle fait appel à des intentions de l'instructeur qui sont parfois difficilement verbalisables et/ou observables. Par exemple sur des objectifs à long terme portant sur la sécurité et/ou l'économique.

Elle présente des formes extrêmement variées selon le contenu, les apprenants, le contexte... mais surtout selon la personnalité, l'expérience et les motivations de l'instructeur.

Elle peut être «en creux ». Par exemple, la rétention d'informations de la part de l'instructeur: ne pas parler d'un matériel concurrent; éviter de conseiller un élève dans un but (éventuellement) pédagogique, afin que celui-ci découvre par lui-même, etc.

Elle ne correspond pas obligatoirement à une démarche spontanée et consciente du pédagogue.

Elle est sensible aux contraintes extrinsèques: économiques, institutionnelles, matérielles, météorologiques, interindividuelles... 

Elle me pose en tant que chercheur des questions méthodologiques et
épistémologiques : certaines méta-chaînes opératoires que j'ai observées résultent de
formations que j'ai dispensées au cours des années précédentes!

\section{L'anthropologie des techniques peut-elle fournir des outils concrets à l'enseignement ?}

Mon implication dans le champ du paramoteur m'a conduit à adopter une position pragmatique, dans un objectif de recherche-action. Décrire, analyser et comprendre... devait aussi me permettre d'apporter du concret aux futurs instructeurs et même à ceux déjà en place, puisqu'une réactualisation des connaissances est obligatoire tous les deux ans, en centre de formation d'instructeurs. Or, la formation paramoteur n'est qu'un cas particulier de la formation continue qui fait l'objet d'une forte demande sociale. Mes incursions dans les domaines des sciences de l'éducation, de la sociologie de l'éducation ou de l'ingénierie de la formation m'ont amené à constater les faits suivants :

La prévalence des contraintes économiques et le peu d'intérêt porté aux facteurs culturels :

«L'incidence forte des outils de gestion, le passage au premier plan des pratiques d'achat, l'entrée en scène de procédures de normalisation et standardisation des démarches, tout cela risque en effet de modifier non seulement les rôles, mais aussi la culture spécifique de la formation continue » (Layole 1994).

Comme les autres formateurs en paramoteur (que ce soit d'instructeurs ou de pilotes) je suis personnellement confronté à ces pressions en tant que responsable d'un organisme de formation qui dépend de l'Aviation civile, ou en tant que membre d'une fédération et même en tant que partie prenante d'un système technique qui comporte des acteurs aux motivations économiques ou politiques fort éloignées de la pédagogie. 


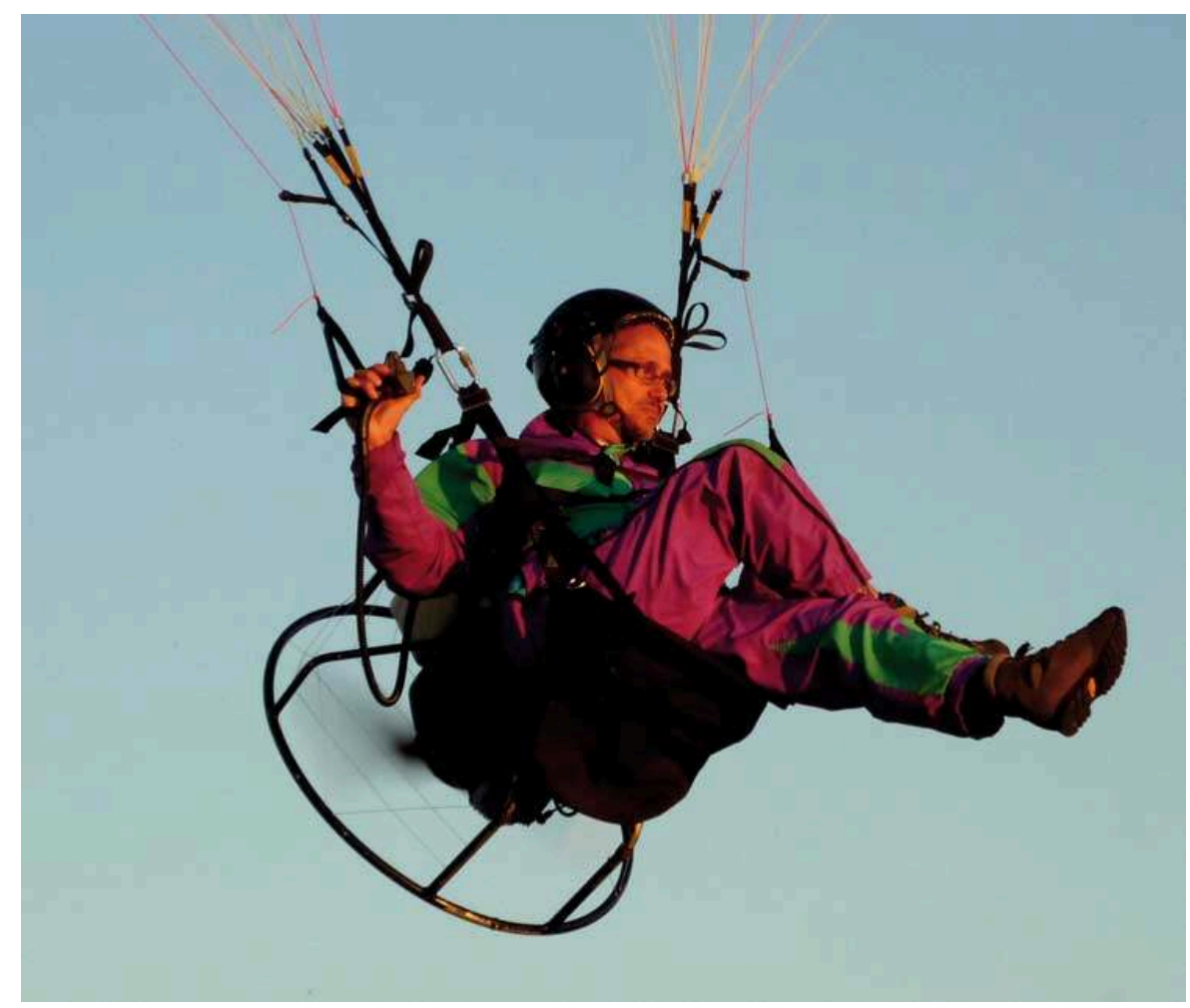

(c) M. Ruet

Les choix techniques (par exemple le port du casque) sont grandement des conséquences de l'apprentissage vicariant et des méta-chaînes opératoires de l'instructeur.

La centration des recherches sur l'apprenant :

« Les styles pédagogiques ont reçu moins d'attention de la part des praticiens et des chercheurs que les styles d'apprentissage. Deux des causes en sont sans doute d'une part la difficulté matérielle à observer les conduites des enseignants sans les modifier par l'observation et d'autre part la réticence, souvent constatée, des enseignants à se prêter à l'observation de leurs pratiques » (Chartier 2003 : 23).

En 2008, la parution de l'ouvrage d'Yves Guégan, Les Ruses éducatives, 100 stratégies pour mobiliser les élèves, m'a apporté une mine de données empiriques et la confirmation que le savoir-faire artisanal $\mathrm{du}$ formateur n'est pas en odeur de sainteté dans l'enseignement "classique », ce que j'avais par ailleurs constaté dans mes recherches bibliographiques sur la formation des formateurs. Pourtant, si cet ouvrage présente un panel étonnant de "ruses » et une riche réflexion épistémologique, il manque encore une méthode de recueil des données qui dépasse la démarche ethnométhodologique du recueil de discours des acteurs.

Enfin, la notion de curriculum cachée ${ }^{29}$ et son rôle dans la transmission culturelle disposent également de bien peu d'outils de recherche.

La formation en paramoteur diffère de la formation scolaire initiale, par la légèreté du curriculum formel : point de "valeurs républicaines » en exergue ${ }^{30}$, pas de public captif, assez grande liberté pour le contenu et les méthodes, absence d'horaires (même quantitativement). Par ailleurs, les caractéristiques de cette pratique induisent des curricula réels très variés: formations courtes d'adultes, concurrence économique, matériels très différents, etc., ce qui peut être découvert par l'étude des chaînes opératoires. En revanche - et les particularités de mon terrain m'ont aidé à le découvrir - le curriculum caché et ses variations locales peuvent partiellement être mis 
en évidence par l'étude des méta-chaînes opératoires du formateur, des prescripteurs, voire des apprenants. Ce qui permet d'envisager la mise au jour de transmissions culturelles « non programmées ».

60 La difficulté d'observation des pratiques d'enseignement et le peu de considération qui leur est accordée font courir le risque d'une déperdition lors de la normalisation et de la standardisation des procédures en formation continue. Ce qui est en jeu, c'est la mètis de celui qui transmet du savoir mais également des normes et des valeurs.

61 Savoir-faire de l'artisan qui agit bien plus qu'il ne théorise, la mètis (cf. Detienne \& Vernant 1989) est restée dans l'ombre de la rationalité discursive du logos. Par exemple, la formation des adultes s'est constituée autour d'une ingénierie qui s'est insuffisamment préoccupée de la didactique professionnelle :

"Mais l'institution de cette ingénierie de la formation, qui est peut-être l'invention spécifique de la formation professionnelle continue dans ses trente ans d'existence instituée, a laissé sur le bord de la route un autre projet, tout aussi important, mais sans doute moins urgent: la constitution d'une ingénierie didactique professionnelle, dont l'objectif est d'utiliser l'analyse du travail pour construire des contenus et des méthodes, visant à la formation des compétences professionnelles [...]» (Pastré 2004 : 465).

« Pourtant, malgré sa centralité, la dimension immergée des savoir-faire [...] n'est devenue que très récemment un objet de recherche. Si les études sur ce thème sont encore rares, c'est sans doute parce que les difficultés méthodologiques sont redoutables mais aussi parce qu'il existe, chez les responsables hiérarchiques en général, une volonté d'ignorer les ajustements opérés par les travailleurs dans l'activité - c'est-à-dire leur intelligence pratique - et, au-delà, de reconnaître leur investissement subjectif dans le travail » (Jobert 2004 : 355).

62 Aux difficultés méthodologiques et aux réticences des responsables hiérarchiques, il faut encore ajouter le "laconisme des experts", que ceux-ci soient artisans ou formateurs :

«[...] ils savent faire, mais ils ne savent plus toujours expliquer comment ils font. C'est probablement une des raisons pour lesquelles l'analyse de l'activité est une entreprise difficile : certains la croient impossible, parce qu'ils se heurtent à ce laconisme des experts » (Pastré 2006 : 116). 


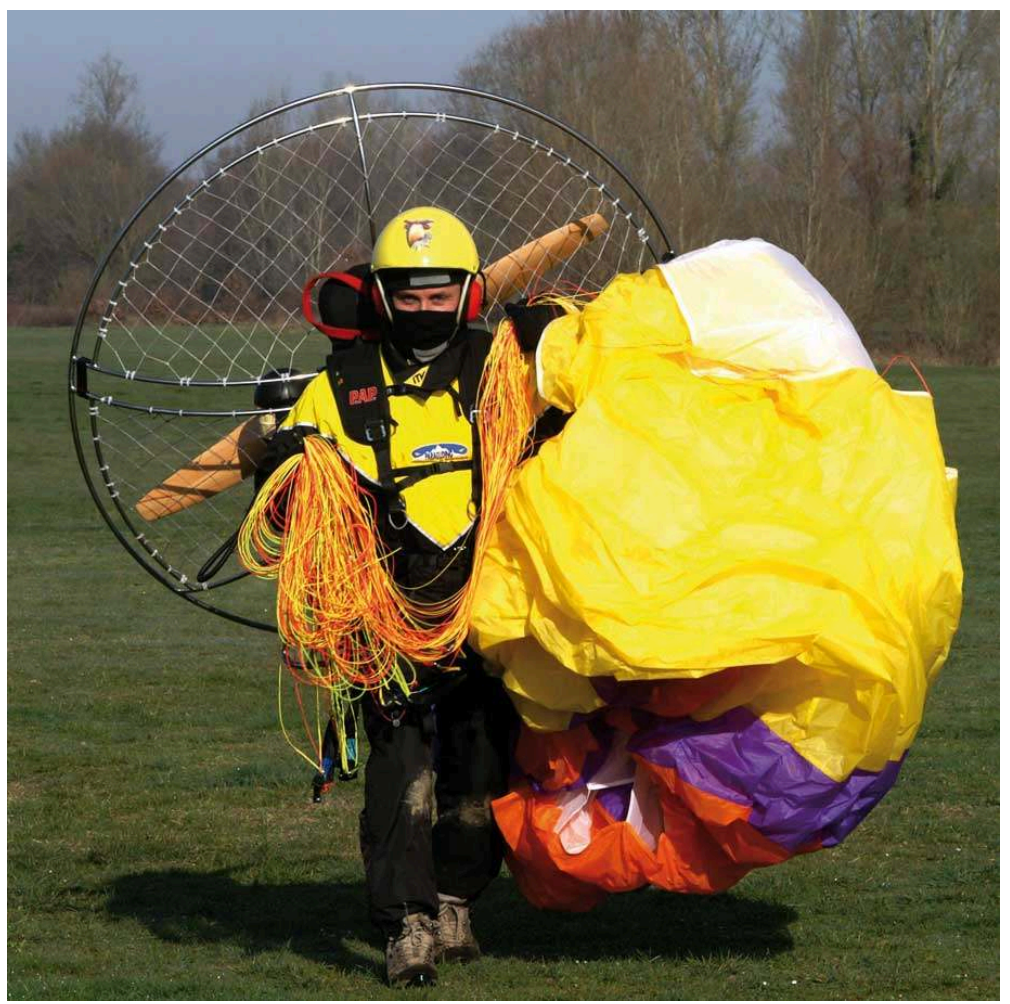

(C) E. Ripoll

À la fin de la formation chacun doit ranger son matériel.

Outil-concept pour le chercheur, le formateur et le formateur de formateurs, la métachaîne opératoire peut être un complément qui va au-delà de la connaissance des «méthodes pédagogiques », des pratiques artisanales ou de celle de l'objet matériel (sa fonction, son usage, son style, ses détournements, etc.).

La mètis de l'instructeur lui permet de transmettre une culture par le biais d'un curriculum caché, hors de portée des institutions. En donnant accès à cette zone d'ombre de la transmission du savoir, l'outil « méta-chaine opératoire " pourrait permettre une distanciation $\mathrm{du}$ formateur par rapport à ses propres pratiques et un regard plus objectif sur les transmissions culturelles et comportementales consécutives de la relation pédagogique.

Loin d'une vision mécaniste de la formation, elle constitue également - au moins sur le terrain du paramoteur - le «Fort Alamo» de l'acteur face aux tentatives de normalisation "par le haut».

\section{BIBLIOGRAPHIE}

Accardo, Alain, 1997, Introduction à une sociologie critique. Bordeaux, Le Mascaret. 
Balfet, Hélène ed., 1991, Observer l'action technique, des chaînes opératoires pour quoi faire ? Paris, CNRS.

Bandura, Albert, 1977, Social Learning Theory. New York, General Learning Press.

Bourdieu, Pierre, 1979, La Distinction, critique sociale du jugement. Paris, Minuit.

Bourdieu, Pierre, 1984, Questions de sociologie. Paris, Minuit.

Chamoux, Marie-Noëlle, 1981, « Les Savoir-faire techniques et leur appropriation : le cas des Nahuas du Mexique ", L'Homme, XXI (3) : 71-94.

Chartier, Daniel, 2003, « Les Styles d'apprentissage : entre flou conceptuel et intérêt pratique ", Savoirs, revue internationale de recherches en éducation et formation des adultes, 2003-2 9-28.

Cresswell, Robert, 1983, " Transferts des techniques et chaînes opératoires ", Techniques \& culture $\mathrm{n}^{\circ} 2$.

Detienne, Marcel, Vernant, Jean-Pierre, 1989, Les Ruses de l'intelligence : La Mètis des Grecs. Paris, Flammarion.

Économie et Statistique, 1996, "Enquête Éducation : l'école, les élèves et les parents ", n² 293, Insee.

Forquin, Jean-Claude, 2008, Sociologie du curriculum. Rennes, PUR.

Guégan, Yves, 2008, Les Ruses éducatives, 100 stratégies pour mobiliser les élèves. Issy-les Moulineaux, ESF.

Jobert, Guy, 2004, « L'Intelligence au travail » in Carré Philippe \& Caspar Pierre eds., Traité des sciences et des techniques de la formation. Paris, Dunod.

Lancry, Alain, 2009, L'Ergonomie. Paris, PUF.

Layole, Gérard, 1994, « Va-t-on vers des révisions déchirantes ? La formation en entreprise », Éducation permanente $\mathrm{n}^{\circ} 118$.

Lemonnier, Pierre, 1976, « La Description des chaînes opératoires : contribution à l'analyse des systèmes techniques », Techniques \& culture, Bulletin $\mathrm{n}^{\circ} 1: 100-151$.

Lemonnier, Pierre, 2004, " Mythiques chaînes opératoires ", Techniques \& culture, [En ligne], 43-44, mis en ligne le 15 avril 2007, Consulté le 3 janvier 2010. URL : http://tc.revues.org/ document1054.html.

Leroi-Gourhan, André, 1943 (1971), Évolution et techniques, L'Homme et la matière. Paris, Albin Michel.

Pastré, Pierre, 2004, « L’Ingénierie didactique professionnelle », in Carre Philippe \& Caspar Pierre eds., Traité des sciences et des techniques de la formation. Paris, Dunod.

Pastré, Pierre, 2006, « Apprendre à faire », in Bourgeois Etienne \& Chapelle Gaëtane eds., Apprendre et faire apprendre. Paris, PUF.

Pelpel, Patrice, 2005, Se former pour enseigner. Paris, Dunod.

Penin, Nicolas, 2006, « Le Sexe du risque », Ethnologie française, XXXVI, 4 : 651-658.

\section{NOTES}

1. Tiré de mon mémoire de master de l'EHESS. 
2. Sont qualifiés ultra-légers motorisés (ULM) les aéronefs monoplaces ou biplaces faiblement motorisés répondant à certaines limitations de masse, de vitesse minimale, de puissance, etc. La réglementation leur concède certains allègements.

3. Les cinq classes d'ULM sont: le paramoteur, le pendulaire, le multi-axes, l'autogire et l'aérostat ULM. Le paramoteur à décollage à pied a pris son essor en 1988.

4. Le terme « Facteurs Humains" provient de l'ergonomie. En aviation, ceux-ci représentent une matière de plus en plus présente lors des examens et que les instructeurs doivent enseigner. Les influences des facteurs biologiques et psychologiques sur les comportements sont - pour le moment - nettement favorisées par rapport aux facteurs sociaux et culturels.

5. Il est difficile d'être précis sur le nombre d'instructeurs paramoteur actifs. Les entrées et sorties de la profession sont nombreuses, certains ont le statut d'indépendants à temps complet, d'autres sont bénévoles ou ont une occupation professionnelle complémentaire, etc.

6. Le savoir-faire algorithmisé est déposé en un support (ouvrage, manuel, fiche, etc.).

7. Les termes «lutte» et «champ » sont empruntés à la sociologie critique (Bourdieu 1984 : 114-120; Accardo 1997 : 43-69).

8. Voir infra: Une «tâche stratégique » de la transmission culturelle.

9. "Mode opératoire " et "mode de réalisation prescrit " sont des formulations qu'utilisent Lancry (2009) et certains ergonomes.

10. Véritable biographie, le carnet de vol accompagne obligatoirement la carrière de l'élèvepilote et du pilote dans les catégories autres que l'ULM. Chaque étape ou qualification y est mentionnée et signée par l'instructeur. Voir les arrêtés du 13 juillet 1998 et du 31 juillet 1981, et en particulier l'article 13.8.1. "Carnet de vol. Le stagiaire ou le titulaire de l'une des licences définies par le présent arrêté, à l'exception toutefois de la licence de pilote d'ULM, doit être détenteur d'un carnet de vol sur lequel sont inscrites la nature et la durée des vols qu'il effectue, au plus tard en fin de journée. Le carnet de vol à jour doit être communiqué sans retard par l'intéressé aux services de contrôle sur simple demande de ceux-ci aux fins de vérification et, en tout cas, au moment de la délivrance ou du renouvellement d'une licence. L'intéressé doit déclarer sur l'honneur que les renseignements portés sur son carnet de vol sont exacts. »

11. Le système est déclaratif. Le propriétaire décrit son appareil sur une fiche d'identification, dans les limites légales de poids et de puissance propres à chaque catégorie d'ULM. Par exemple, pour un paramoteur monoplace de catégorie $1 \mathrm{~A}$ : la puissance maximale continue est inférieure ou égale à $25 \mathrm{~kW}$ et la puissance maximale est inférieure ou égale à $30 \mathrm{~kW}$; la masse maximale est inférieure ou égale à $170 \mathrm{~kg}$; la charge alaire à la masse maximale est inférieure à $30 \mathrm{~kg} / \mathrm{m}^{2}$.

12. Selon lequel un processus cognitif est le résultat d'une réponse immédiate à un stimulus, sans considération de l'environnement. La théorie sociale cognitive estime au contraire que prédomine l'imitation des comportements d'autres individus (apprentissage vicariant).

13. Les pionniers payent souvent très cher le manque de modèles : « Des sacrifices doivent être faits » (Otto Lilienthal, précurseur de l'aviation, après son dramatique accident en 1896).

14. Mais pas uniquement dans un but de rentabilité : la clientèle a tendance à préférer les écoles qui proposent des formations courtes présentant le paramoteur comme une «mobylette de l'air ». L'instructeur qui souhaite former ses élèves sur le long terme doit user de stratégies... qu'il est possible de mettre en évidence par l'étude de méta-chaînes opératoires.

15. Quelques surnoms d'instructeur : « Tonton », « Gourou », « Papa », « El Jefe », « Maître »...

16. Et ce, sur le long terme, comme instructeur.

17. J'ai formé une bonne partie de la profession des instructeurs et j'ai progressivement pris conscience que je leur enseigne depuis longtemps des méta-chaînes opératoires.

18. Voir par exemple : Numéro spécial d'Économie et Statistique 1996, ou Bourdieu 1979.

19. ... mais parfois en totale opposition avec ce qui est demandé à l'élève ! Voir infra.

20. Les trims sont un dispositif permettant de modifier le calage et/ou le profil de l'aile. 
21. L'instructeur, à quelques mètres face à l'élève, donne un signal quand il estime que les meilleures conditions sont réunies (vent, autres pilotes dans la zone, etc.). Le paramoteur étant encombrant et l'aspiration de l'hélice gênant l'observation des variations du vent, cette pratique est même fréquente entre pilotes confirmés. Le «top gonflage » peut être transmis par radio ou par gestes plus ou moins codifiés, et parfois avec des accessoires comme les raquettes, qu'utilisent également le personnel de piste sur les aéroports ou les porte-avions.

22. En paramoteur, et a fortiori en école, on vole surtout le matin tôt et le soir, quand les conditions aérologiques sont calmes.

23. Sur les tâches stratégiques, voir Lemonnier 1976.

24. Puissance appliquée, sens de rotation, type d'hélice, etc., génèrent des effets non désirés en tangage, roulis et lacet. Sommairement, plus le moteur est puissant, plus les effets sont marqués. 25. Avec des choix techniques parfois opposés. Il est par exemple tout à fait possible de prôner la sécurité avec des modèles $\mathrm{A}$, en usant d'autres arguments, et de méta-chaînes opératoires adaptées.

26. ...ou « en amont », dans une perspective structuro-constructiviste.

27. Et là, on se retrouve « en amont » du choix technique!

28. Entretien avec un jeune pompier débutant en paramoteur.

29. Courant de recherche qui s'est développé depuis les années 70 , la sociologie du curriculum étudie la construction sociale des savoirs scolaires. Selon cette approche, l'institution propose ou impose un curriculum formel (sommairement, le programme et les méthodes). Le formateur effectue un curriculum réel (sommairement, les choix, la forme et le fond de ce qui se passe en cours). Le curriculum caché représente tout ce qui est transmis sans être formellement exprimé (en particulier au niveau des valeurs). Le curriculum caché est présent dans les deux premiers (idéologie par exemple), mais il est également le fait des apprenants eux-mêmes (langage, normes non-institutionnelles, valeurs, etc.). Voir Forquin 2008.

30. "Le professeur des écoles est un fonctionnaire porteur des valeurs de la République»: première phrase du préambule du «Référentiel des compétences et capacités caractéristiques d'un professeur des écoles », cité par Pelpel $2005: 369$.

\section{RÉSUMÉS}

Méta-chaîne opératoire et transmission culturelle. Le paramoteur est le plus petit des ULM et se caractérise par la polyvalence des formateurs, la variabilité des modes opératoires et de celle des objets matériels de la pratique. L'apprentissage a lieu en groupe et l'observation de l'autre y tient une place importante. L'instructeur-revendeur est un prescripteur de matériels, de pratiques et d'adhésions aux valeurs de fabricants, clubs, fédérations, forums Internet, etc. Il subit des pressions météorologiques, sociales et psychologiques. Enjeu de luttes politiques et économiques, la formation des pilotes et des instructeurs fait l'objet de tentatives de normalisation. Au-delà de l'efficacité de l'action sur la matière les rapports qu'entretiennent le social et le technique peuvent être observés au cours de la formation au pilotage, par l'étude des chaînes opératoires. Cependant, le chercheur - impliqué sur un très long terme - a pu mettre au jour des "métachaînes opératoires" fugaces, variables, mêlant des aspects techniques, pédagogiques et économiques. Par leur influence déterminante sur les comportements à long terme des pratiquants, ces méta-chaînes opératoires sont les vecteurs privilégiés de la transmission d'une 
culture de la sécurité. Elles sont pourtant difficiles à observer, à quantifier et à normaliser. Décrivant le savoir-faire artisanal du formateur - sa mètis - l'outil-concept «méta-chaîne opératoire " permet de mieux cerner cette zone d'ombre de la transmission du savoir et son rôle dans la sécurité des pratiquants. Dans une démarche plus générale, l'étude des méta-chaînes opératoires pourrait également apporter une aide méthodologique à l'ingénierie de la formation ou à la sociologie du curriculum et valoriser le savoir-faire de l'acteur face aux normes édictées par les institutions.

Meta-operational Sequence and Cultural Transmission. Paramotor is the smallest micro-light aircraft and is characterized by the versatility of the instructor, the changeability of the operationnal sequences and of the material objects of practice. Learning takes place in a group and observing others one holds an important place. The instructor-seller is a prescriber of equipments, practices and values of manufacturers, clubs, confederations, Internet forums, etc. It is subjected to meteorological, social, psychological pressures. Training of the pilots and the instructors is a stake of political and economic conflicts, even whitin trials of normalization. Beyond effective action on material, reports between social and technics can be noticed in the course of training, by the study of operationnal sequences. However, the researcher - implicated on very long-term - can discover "meta operationnal sequences", which are fleeting and variable, blending technical, pedagogic and economic aspects. By their decisive influence on the long-term behaviours of the pilots, these meta operationnal sequences are the privileged vectors of the transmission of a culture of security. They are however difficult to notice, to quantify and to normalize. Describing the craft know-how of the instructor, the tool and concept «meta operationnal sequences » allows to fathom this black hole of the transmission of knowledge and its role in the security of the pilots. In a more general step, the study of operationnal sequences can also bring a methodological help to the instructional Design or to the sociology of the curriculum, and promote the know-how of the actor facing norms issued by institutions.

\section{INDEX}

Keywords : apprentissage vicariant, craft know-how, ingénierie de la formation, instructional design, métacognition, metacognition, normalisation, normalization, savoir-faire artisanal, transmission du savoir, Transmission of knowledge, vicariant learning

\section{AUTEUR}

\section{ERIC RIPOLL}

